\title{
Worldsheet descriptions of wrapped NS five-branes
}

\author{
Kentaro Hori \\ Institute for Advanced Study \\ Princeton, NJ 08540, USA \\ E-mail: hori@ias.edu

\section{Anton Kapustin} \\ California Institute of Technology
Pasadena, CA 91125, USA
E-mail: kapustin@theory.caltech.edu
}

ABSTRACT: We provide a world-sheet description of Neveu-Schwarz five-branes wrapped on a complex projective space. It is an orbifold of the product of an $\mathcal{N}=2$ minimal model and the IR fixed point of a certain linear sigma model. We show how the naked singularity in the supergravity description is resolved by the world-sheet CFT. Applying mirror symmetry, we show that the low-energy theory of NS5-branes wrapped on $\mathbb{C P}^{1}$ in Eguchi-Hanson space is described by the Seiberg-Witten prepotential for $\mathcal{N}=2$ super-Yang-Mills, with the gauge group given by the ADE-type of the five-brane. The world-sheet CFT is generically regular, but singularities develop precisely at the Argyres-Douglas points and massless monopole points of the space-time theory. We also study the low-energy theory of NS5branes wrapped on $\mathbb{C P}^{2}$ in a Calabi-Yau 3 -fold and its relation to $(2,2)$ super-Yang-Mills theory in two dimensions.

Keywords: p-branes, String Duality, Conformal Field Models in String Theory, 


\section{Contents}

1. Introduction and summary 1

2. Five-branes wrapped on $\mathbb{C P}^{n}$

3. Linear sigma model and its infrared limit 6

4. T-Dual background and wrapped branes 10

5. Mirror description of wrapped five-branes 13

6. Non-perturbative 4d physics from the world-sheet 16

6.1 Derivation of the Seiberg-Witten solution

6.2 Argyres-Douglas points 18

6.3 Supergravity description of the coulomb branch

7. Compactification on $\mathbb{C P}^{2}$

8. Relation to Super-Yang-Mills theories 22

9. Concluding remarks 25

\section{Introduction and summary}

Finding string theory duals of gauge theories or gauge theory duals of strings has been regarded as an important problem since the work of 't Hooft [1. AdS/CFT duality is an important development in this direction. However, in this case the world-sheet CFT is usually not under control and one has to work in the supergravity approximation. Furthermore, one is usually restricted to conformally invariant and supersymmetric theories. In particular, we still do not know the string dual of pure Yang-Mills in $3+1$ dimensions. It was suggested in ref. [2] that the string dual of such a theory should be some non-critical string where the Liouville field represents the renormalization scale.

There is one interesting example of a non-scale-invariant theory which has a string theory dual with a controllable worldsheet description: the decoupled theory on NeveuSchwarz five-branes [3]. When NS5-branes are coincident, the supergravity solution develops an infinite "throat" with a linearly growing dilaton [4. It has been proposed that the theory of strings propagating in the throat is dual to the Little String Theory living on the five-branes 5 . The corresponding world-sheet CFT is exactly soluble and can be used to find the spectrum of the theory. However, it is not suitable for computing correlators 
because the dilaton grows without bound as one moves toward the branes. When the fivebranes are separated, the system is still described by the exactly solvable CFT [6]:

$$
\mathcal{C}_{m}^{(1)}=\left[\frac{\mathrm{SL}(2, \mathbb{R})_{m+2}}{\mathrm{U}(1)} \times \frac{\mathrm{SU}(2)_{m-2}}{\mathrm{U}(1)}\right] / \mathbb{Z}_{m}
$$

where $m$ is the number of five-branes. $\mathrm{SL}(2, \mathbb{R})_{m+2} / \mathrm{U}(1)$ is the euclidean $2 \mathrm{~d}$ black hole background which has a semi-infinite cigar geometry with an asymptotically linear dilaton [7], while $\mathrm{SU}(2)_{m-2} / \mathrm{U}(1)$ stands for the $\mathcal{N}=2$ minimal model. In this case the dilaton is bounded (its maximal value is achieved at the tip of the cigar), and one can hope that string perturbation theory is sufficient. This approach gives interesting results about the physics of LSTs at finite $m$ [8, 9, 10].

Upon compactification LSTs can flow to a number of supersymmetric gauge theories in lower dimensions. For example, upon compactification on $\mathbb{C P}^{1}$ type-IIB LST flows either to an $\mathcal{N}=2$ or $\mathcal{N}=1$ super-Yang-Mills theory in $d=4$, depending on the "twist." The twist describes the geometry of the normal bundle; for example, when the $\mathbb{C P}^{1}$ is the vanishing cycle of the Eguchi-Hanson space, one obtains $\mathcal{N}=2$ supersymmetry in $3+1$ dimensions. Clearly, it is of interest to understand the corresponding superstring backgrounds. The supergravity solutions describing the near horizon geometries of such wrapped five-branes have been studied in [11-15]. The solution corresponding to $\mathcal{N}=1$ super-Yang-Mills turns out non-singular and exhibits a spontaneous breaking of R-symmetry [11]. On the other hand, the supergravity solution corresponding to $\mathcal{N}=2$ super-Yang-Mills has a naked singularity [12, 13].

In this paper, we provide a regular world-sheet description of the $\mathcal{N}=2$ compactification. The world-sheet theory is very similar to that for flat separated five-branes, except that the two-dimensional black hole in (1.1) is replaced by a four-dimensional dilatonic background parametrized by $m$. That is, the world-sheet CFT for $m$ five-branes wrapped on $\mathbb{C P}^{1}$ is of the form

$$
\mathcal{C}_{m}^{(2)}=\left[4 \mathrm{~d} \text { background }_{m} \times \frac{\mathrm{SU}(2)_{m-2}}{\mathrm{U}(1)}\right] / \mathbb{Z}_{m}
$$

The 4d background is the one found in ref. [16] by solving the one-loop beta-function equations. It has a $\mathrm{U}(2)$-invariant Kähler metric and a dilaton which is asymptotically linear but is bounded from above. The system $\mathcal{C}_{m}^{(2)}$ is a well-defined CFT, whose only peculiarities come from the non-compactness of the target space. We will see that a simple T-duality converts it to the supergravity solution of refs. [12, 13] describing five-branes wrapped on $\mathbb{C P}^{1}$. The naked singularity of the latter is thus resolved by the world-sheet CFT. In fact, one can describe the system without resort to the one-loop approximation: the $4 \mathrm{~d}$ background can be defined as the infra-red limit of a gauged linear sigma model (LSM) which is a simple generalization of the LSM that yields the $2 \mathrm{~d}$ black hole [17.

The supergravity solution of refs. [12, 13] actually has one parameter $k$ which can take any value from -1 to $+\infty$. It was argued to correspond to a Coulomb branch modulus, with the origin of the Coulomb branch corresponding to $k=-1$. Likewise, we are able to construct a one-parameter family of LSMs which corresponds to this one-parameter family 
of supergravity solutions. Thus for any $k$ the naked singularity is resolved by world-sheet CFT. There is one exception though: at a single point on this line the world-sheet CFT becomes singular. Using the mirror description (see below), we show that at the singularity the theory on the five-branes flows to an $\mathcal{N}=2 d=4$ SCFT of the Argyres-Douglas type.

Unlike the euclidean $2 \mathrm{~d}$ black hole, its $4 \mathrm{~d}$ analogue does not appear to be integrable. Nevertheless, when the number of five-branes $m$ is large, it is weakly curved everywhere, and thus all scattering amplitudes can, in principle, be computed order by order in $1 / \mathrm{m}$ expansion. Combined with the exactly known correlators of the minimal model, they provide a complete holographic description of five-branes wrapped on $\mathbb{C P}^{1}$.

Linear sigma models that appear in this paper have mirror duals of the Liouville type [18, 17]. Combined with the fact that the orbifold CFT $\frac{\mathrm{SU}(2)_{m-2}}{\mathrm{U}(1)} / \mathbb{Z}_{m}$ is mirror to the LG model with the superpotential $W=X^{m}$, this allows us to find the mirror descriptions of the above CFTs. The mirror of the CFT (1.1) is the Landau-Ginzburg orbifold

$$
\mathcal{C}_{m}^{(1)} \stackrel{\text { mirror }}{=}\left[W=\mathrm{e}^{-m Z}+X^{m}\right] / \mathbb{Z}_{m}
$$

where $\mathbb{Z}_{m}$ action is generated by $Z \rightarrow Z-\frac{2 \pi i}{m}$ and $X \rightarrow \mathrm{e}^{\frac{2 \pi i}{m}} X$. Moving NS5-branes corresponds to deforming the superpotential by adding the terms $\mathrm{e}^{-(m-\ell) Z} X^{\ell}, \ell=0,1, \ldots, m-2$. The relation (1.3) entails the relation between NS5-branes and ALE spaces proposed in [6] . The family of deformations $W=\mu \mathrm{e}^{-m Z}+X^{m}$ contains a singular point $\mu=0$ which corresponds to coincident NS5-branes, or equivalently the singular ALE space.

The mirror of the CFT (1.2) for NS5-branes wrapped on $\mathbb{C P}^{1}$ is

$$
\mathcal{C}_{m}^{(2)} \stackrel{\text { mirror }}{=}\left[W=\mathrm{e}^{-m Z}\left(\mathrm{e}^{-Y}+\mathrm{e}^{Y}\right)+X^{m}\right] / \mathbb{Z}_{m},
$$

where $\mathbb{Z}_{m}$ acts on $Z$ and $X$ in the same way as above and leaves $Y$ invariant. Moving on the Coulomb branch of the five-brane theory again corresponds to adding the terms $\mathrm{e}^{-(m-\ell) Z} X^{\ell}, \ell=0,1, \ldots, m-2$. Using this mirror description, one can reproduce the Seiberg-Witten solution of the low energy $\mathcal{N}=2$ super-Yang-Mills theory. The family of deformations $W=\mathrm{e}^{-m Z}\left(\mathrm{e}^{-Y}+\mathrm{e}^{Y}+u_{0}\right)+X^{m}$ corresponds to the one parameter family of supergravity solutions mentioned above. We will see that the world-sheet theory is singular at $u_{0}= \pm 2$. From the point of view of low-energy super-Yang-Mills theory, this is the Argyres-Douglas point. As in the case of the conifold singularity, the breakdown of the world-sheet CFT signals non-trivial infrared physics in target space.

In this paper, we will also consider a compactification of LST on $\mathbb{C P}^{2}$ which preserves $(2,2)$ supersymmetry in $1+1$ dimensions. This corresponds to NS5-branes wrapped on a $\mathbb{C P}^{2}$ in a non-compact Calabi-Yau 3-fold, the total space of the line bundle $\mathcal{O}(-3)$ over $\mathbb{C P}^{2}$. The world-sheet $\mathrm{CFT}$ is of the form

$$
\mathcal{C}_{m}^{(3)}=\left[6 \text { background }_{m} \times \frac{\mathrm{SU}(2)_{m-2}}{\mathrm{U}(1)}\right] / \mathbb{Z}_{m}
$$

where the $6 \mathrm{~d}$ background can be described as a U(3)-invariant dilatonic solution of the one-loop beta function equations for large $m$, or as the infra-red limit of a simple LSM for any $m$. The LSM realization enables us to find the mirror theory, which is closely related to 
the world-sheet CFT of a certain non-compact Calabi-Yau 4-fold. The mirror description enables us to compute world-sheet instanton correction to the space-time superpotential.

In the infrared, LSTs compactified on complex projective spaces are in the same universality class as super-Yang-Mills theories in the remaining flat dimensions, but are not quite the same as the latter. One could hope to find some limit where extra degrees of freedom decouple and one obtains exactly super-Yang-Mills. Unfortunately, we find that in the decoupling limit the string coupling should be taken large, both for $\mathbb{C P}^{1}$ and $\mathbb{C P}^{2}$ compactifications. Thus our worldsheet descriptions are applicable only when the compactified LST is quite different from the super-Yang-Mills theory. However, in the $\mathbb{C P}^{1}$ case, due to a supersymmetric non-renormalization theorem, the low energy prepotential does not depend on the string coupling, and we recover the correct solution of the super-Yang-Mills theory. In fact, world-sheet intantons precisely correspond to Yang-Mills instantons, which are responsible for non-perturbative corrections to the prepotential. On the other hand, for the $\mathbb{C P}^{2}$ compactification we argue that and world-sheet instanton contributions to the space-time (twisted) superpotential vanish in the decoupling limit.

The paper is organized as follows. In section 2 we review "twisted" compactifications of five-branes on $\mathbb{C P}^{n}$ and their symmetries. In section 3 we define linear sigma models of interest to us and study their infrared fixed points in world-sheet perturbation theory. In section 4 we relate these linear sigma models to wrapped five-branes. In section 0 we find the mirror description of our world-sheet CFTs in terms of Landau-Ginzburg models. In section 6 we use this mirror description to determine the Seiberg-Witten prepotential for five-branes wrapped on $\mathbb{C P}^{1}$ and investigate the world-sheet interpretation of ArgyresDouglas points. We also obtain the supergravity solution describing five-branes away from the origin of the Coulomb branch by studying the RG flow on the world-sheet. In section 7 we perform a similar analysis for five-branes wrapped on $\mathbb{C P}^{2}$. Section 8 discusses the decoupling limit in which the theory of wrapped five-branes reduces to super-Yang-Mills. We conclude with remarks about the (lack of) integrability of the world-sheet CFT, connection with non-critical superstrings, and other issues.

Throughout this paper we set $\alpha^{\prime}=1$. In particular, the gauge coupling of the low energy Yang-Mills theory on NS5-branes is given by $g_{6}^{2}=2(2 \pi)^{3}$.

\section{Five-branes wrapped on $\mathbb{C P}^{n}$}

Type IIB NS5-brane configurations in flat space which preserve sixteen supercharges have an ADE classification. Simply putting $m$ five-branes on top of each other yields a configuration of type $A_{m-1}$. If $m$ is even, then putting $m / 2$ five-branes on top of an $O N^{-}$ six-plane yields a configuration of type $D_{\frac{m}{2}+1}$. We remind that an $O N^{-}$plane is defined as an orbifold of type-IIB string by the symmetry which reflects four of the coordinates and in addition multiplies all fields by $(-1)^{F_{L}}$, where $F_{L}$ is the left-moving fermion number. The near-horizon limit is given by the Callan-Harvey-Strominger background [ $⿴$ ]

$$
\mathbb{R}^{5+1} \times \mathbb{R} \times \mathbb{S}^{3}
$$


where the dilaton depends linearly on the affine coordinate on $\mathbb{R}$ and there is an H-flux through $\mathbb{S}^{3}$. Thus the $\mathbb{S}^{3}$ part is described by an SU(2) WZW model. The ADE classification of five-branes originates from the ADE classification of affine $\mathrm{SU}(2)$ modular invariants. Each such brane configuration gives rise to a Little String Theory with sixteen supercharges in $d=6$. The $\mathrm{SU}(2)_{\ell} \times \mathrm{SU}(2)_{r}$ current algebra of the $\mathrm{SU}(2)$ WZW model gives rise to the R-symmetry group of the LST. Under the $\mathrm{SO}(1,5) \times \mathrm{SU}(2)_{\ell} \times \mathrm{SU}(2)_{r}$ bosonic symmetry the supercharges transform as $\left(\mathbf{4}_{+}, \mathbf{2}, \mathbf{1}\right)+\left(\mathbf{4}_{-}, \mathbf{1}, \mathbf{2}\right)$, and satisfy a reality condition.

When compactifying on $\mathbb{C P}^{1}$, we can "twist" the theory by embedding the U(1) structure group of the $\mathbb{C P}^{1}$ spin bundle into the R-symmetry group. We choose to embed $\mathrm{U}(1)$ diagonally into the $\mathrm{U}(1)_{\ell} \times \mathrm{U}(1)_{r}$ subgroup of the $\mathrm{SU}(2)_{\ell} \times \mathrm{SU}(2)_{r}$ R-symmetry. In this case each of the two supercharge multiplets in the $d=6$ theory yields a complex supercharge in $d=4$. Therefore we end up with an $\mathcal{N}=2$ theory in $d=4$. The $\mathrm{U}(1)$ part of the $\mathrm{SU}(2) \times \mathrm{U}(1)$ R-symmetry group is the $\mathrm{U}(1)$ which is off-diagonally embedded into $\mathrm{U}(1)_{\ell} \times \mathrm{U}(1)_{r}$. The $\mathrm{SU}(2)$ R-symmetry of the low-energy theory is not manifest, except the $\mathrm{U}(1)$ subgroup which can be identified as the diagonal of $\mathrm{U}(1)_{\ell} \times \mathrm{U}(1)_{r}$. We note that the $d=6$ R-symmetry group $\mathrm{SU}(2)_{\ell} \times \mathrm{SU}(2)_{r}$ acts on the group element of the $\mathrm{SU}(2) \mathrm{WZW}$ model as $g \mapsto g_{\ell} g g_{r}^{-1}$. Thus the $\mathrm{U}(1)$ R-symmetry group in $d=4$ acts as follows: ${ }^{1}$

$$
\mathrm{U}(1)_{R}: g \mapsto \mathrm{e}^{i \alpha} g \mathrm{e}^{i \alpha},
$$

while the action of the $\mathrm{U}(1)$ subgroup used in the twisting is

$$
\mathrm{U}(1)_{\text {twist }}: g \mapsto \mathrm{e}^{i \gamma} g \mathrm{e}^{-i \gamma} .
$$

This twist is the same as in ref. [12, 13]. ${ }^{2}$ As explained in ref. [12], it corresponds to five-branes wrapped on the holomorphic 2-cycle of the Eguchi-Hanson space.

In the low-energy limit an LST of type $A, D$, or $E$ reduces to $\mathcal{N}=2 d=6$ superYang-Mills theory with a simply laced gauge group of the appropriate type. It is natural to expect that its "twisted" Kaluza-Klein reduction on $\mathbb{C P}^{1}$ yields $\mathcal{N}=2 d=4$ super-YangMills. Indeed, it is clear that we get an $\mathcal{N}=2$ vector multiplet in $d=4$. There is a single complex scalar in such a multiplet. On the other hand, the scalars in $d=6$ transform as a $(2,2)$ of the R-symmetry group, and therefore upon Kaluza-Klein reduction they yield precisely one complex scalar. This leaves no room for any hypermultiplets.

Similarly, we can consider a twisted compactification of type-IIB LST on $\mathbb{C P}^{2}$. Although $\mathbb{C P}^{2}$ does not admit a spin structure, there is a twisted compactification that is well defined and preserves four supercharges. We recall that the tangent bundle of a Kähler surface has a reduced structure group $\mathrm{U}(2) \subset \mathrm{SO}(4)$, and its lift to the spin group $\mathrm{SU}(2) \times \mathrm{SU}(2)$ is $\mathrm{SU}(2) \times \mathrm{U}(1)$. The latter group covers $\mathrm{U}(2)$ twice, with kernel $(-1,-1)$. The twisting we choose is to embed the spinor group $\mathrm{SU}(2) \times \mathrm{U}(1)$ into the $\mathrm{SU}(2)_{\ell} \times \mathrm{SU}(2)_{r}$ R-symmetry group, so that the $\mathrm{SU}(2)$ factor is embedded trivially, while the $\mathrm{U}(1)$ factor is embedded diagonally into $\mathrm{U}(1)_{\ell} \times \mathrm{U}(1)_{r}$. Then the $d=6$ supercharges transform vectorially under the twisted $\mathrm{SU}(2) \times \mathrm{U}(1)$ action - the kernel $(-1,-1)$ acts trivially. In other

\footnotetext{
${ }^{1}$ We realize $\mathrm{SU}(2)$ as the group of unit quaternions.

${ }^{2}$ The twist used in 11] for $\mathcal{N}=1$ compactification on $\mathbb{C P}^{1}$ is $g \mapsto \mathrm{e}^{i \gamma} g$.
} 
words, the modified spin group is $\mathrm{U}(2)$. Under this modified spin group, the supercharges transform as follows:

$$
\begin{aligned}
& \left(\mathbf{4}_{+}, \mathbf{2}, \mathbf{1}\right)=\mathbf{2}_{+}+\overline{\mathbf{2}}_{+}+\operatorname{det}_{-}+\overline{\operatorname{det}}_{-}+\mathbf{1}_{-}+\mathbf{1}_{-} \\
& \left(\mathbf{4}_{-}, \mathbf{1}, \mathbf{2}\right)=\mathbf{2}_{-}+\overline{\mathbf{2}}_{-}+\operatorname{det}_{+}+\overline{\operatorname{det}}_{+}+\mathbf{1}_{+}+\mathbf{1}_{+}
\end{aligned}
$$

On the right hand side, $\mathbf{2}, \overline{\mathbf{2}}$, det, $\overline{\mathrm{det}}, \mathbf{1}$ are the fundamental, the anti-fundamental, determinant, anti-determinant, and trivial representations of $\mathrm{U}(2)$ respectively (the subscripts \pm show the chirality in the uncompactified $1+1$ dimensions). In particular, supercurrents are sections of well-defined vector bundles on $\mathbb{C P}^{2}$.

To prove that this twisted compactification is well-defined, we need to show that all fields of the LST transform vectorially under the twisted spinor group. Since the supercharges are well-defined as we have seen, it is enough to show that bosons transform vectorially. This is equivalent to showing that bosons transfom vectorially under the $\mathrm{SU}(2)_{\ell} \times \mathrm{SU}(2)_{r} \mathrm{R}$-symmetry. In other words, we need to check that $j_{\ell}+j_{r} \in \mathbb{Z}$. The spectrum of LST can be found using the holographic description [5. In particular, the $\mathrm{SU}(2)_{\ell} \times \mathrm{SU}(2)_{r}$ spins for bosons are the same as in the $\mathrm{SU}(2)$ WZW model up to integer shifts. For all ADE modular invariants, the condition $j_{L}+j_{R} \in \mathbb{Z}$ is satisfied [19]. This completes the proof.

Since the holonomy group of $\mathbb{C P}^{2}$ is the entire U(2), eq. (2.4) shows that there are only four covariantly constant spinors on $\mathbb{C P}^{2}$ - two $\mathbf{1}_{+}$'s and two $\mathbf{1}_{-}$'s. Thus we end up with $(2,2)$ supersymmetric field theory in $1+1$ dimensions. The $\mathrm{U}(1)_{\ell}$ and $\mathrm{U}(1)_{r} \mathrm{R}$-symmetries descend to the left and right $\mathrm{R}$-symmetries of the $(2,2)$ algebra. It is also easy to see that the twisted Kaluza-Klein reduction of an $\mathcal{N}=(1,1) d=6$ vector multiplet yields a single $(2,2)$ vector multiplet in $d=2$. Thus the naïve dimensional reduction of $(1,1)$ LST yields $(2,2)$ super-Yang-Mills theory in $1+1$ dimensions, with an ADE gauge group.

Note that this twist corresponds to $\mathbb{C P}^{2}$ being an exceptional divisor in a Calabi-Yau 3-fold. The local model for this is an $O(-3)$ bundle over $\mathbb{C P}^{2}$. There exists an ALE Ricci flat Kähler metric on this manifold which generalizes the Eguchi-Hanson metric [20], but we will not need its explicit form here. Another way to think about this non-compact Calabi-Yau is as a crepant resolution of the $\mathbb{C}^{3} / \mathbb{Z}_{3}$ orbifold singularity [20].

To decouple Kaluza-Klein modes one must take the size of the compactification manifold to be small. Since LSTs are non-local theories, it is not completely clear what this means. In other words, it is not obvious in what regime one can simultaneously decouple the massive Kaluza-Klein modes and approximate the LST by $d=6$ super-Yang-Mills. This issue is discussed in more detail in section 8.

\section{Linear sigma model and its infrared limit}

In this section we study a certain gauged linear sigma model. In the next section, we will tensor it with an $\mathcal{N}=2$ minimal model and show that it is T-dual to a configuration of wrapped five-branes. 
We follow the notations and conventions of ref. [17]. We consider an $\mathcal{N}=2$ LSM with the following superspace lagrangian:

$$
S=\frac{1}{2 \pi} \int d^{2} x d^{4} \theta\left[\sum_{i=1}^{n} \Phi_{i}^{\dagger} e^{V} \Phi_{i}+\frac{k}{4}(P+\bar{P}+V)^{2}-\frac{1}{2 e^{2}} \Sigma^{2}\right]
$$

Here $P$ and $\Phi_{i}, i=1, \ldots, n$, are chiral superfields, $V$ is an abelian vector superfield, and $\Sigma=\bar{D}_{+} D_{-} V$. Under $\mathrm{U}(1)$ gauge transformations the fields transform as $\Phi_{i} \rightarrow \mathrm{e}^{i \Lambda} \Phi_{i}$, $P \rightarrow P+i \Lambda$ and $V \rightarrow V-i \Lambda+i \bar{\Lambda}$, where $\Lambda$ is a chiral superfield. The imaginary part of $P$ is periodically identified with period $2 \pi$.

The chiral field $P$ can be dualized to a twisted chiral superfield $Y_{P}$. In terms of $Y_{P}, \Phi_{i}$, and $V$ the lagrangian takes the form

$$
S=\frac{1}{2 \pi} \int d^{2} x d^{4} \theta\left[\sum_{i=1}^{n} \Phi_{i}^{\dagger} e^{V} \Phi_{i}-\frac{1}{2 k}\left|Y_{P}\right|^{2}-\frac{1}{2 e^{2}} \Sigma^{2}\right]+\frac{1}{4 \pi}\left(\int d^{2} x d^{2} \tilde{\theta} \Sigma Y_{P}+\text { h.c. }\right) .
$$

This $\mathcal{N}=2$ gauge theory has a non-anomalous axial $\mathrm{U}(1)_{R}$ symmetry, therefore in the infrared the theory flows to a non-trivial CFT. The central charge of this CFT has been computed in ref. [17:

$$
c_{I R}=3 n\left(1+\frac{2 n}{k}\right) .
$$

This result is exact. For large $k$ the CFT is weakly coupled and can be represented by an $\mathcal{N}=2$ supersymmetric non-linear sigma model with a $2 n$-dimensional target space. Since there is no $H$-field in this CFT, the target space must be kählerian.

Our goal in this section is to determine this kählerian metric, at least for large $k$, where one can use sigma-model perturbation theory. Following ref. [17], we do it in two steps. On the first step, we integrate out the gauge fields classically, which is a good approximation for large $k$. This results in a non-linear sigma model with a $2 n$-dimensional kählerian target space. This target space, however, does not solve the beta-function equations. On the second step, we find the kählerian $2 n$-dimensional metric which solves the beta-function equations and has the same symmetries and asymptotics as the metric found in step one. Since such a metric is unique, the LSM must flow to the non-linear sigma-model with this metric. Note that the one-loop beta function equations are sufficient because both the initial and final kählerian metrics are weakly curved for large $k$.

Step 1. In principle, one could integrate out the vector multiplet in superspace. However, the resulting Kähler potential is not expressible in elementary functions, and therefore we prefer to work with component fields. For simplicity, we provide a detailed derivation for the case $n=2$ and then write down the answer in the general case.

The kinetic energy for the bosonic matter fields is encoded in the following metric:

$$
\mathrm{d} s^{2}=2\left|\mathrm{~d} \Phi_{1}\right|^{2}+2\left|\mathrm{~d} \Phi_{2}\right|^{2}+k|\mathrm{~d} P|^{2} .
$$

It is convenient to parametrize $\Phi_{i}$ as follows:

$$
\begin{aligned}
& \Phi_{1}=r e^{\frac{i}{2}(\psi-\phi)} \cos \frac{\theta}{2}, \\
& \Phi_{2}=r e^{\frac{i}{2}(\psi+\phi)} \sin \frac{\theta}{2},
\end{aligned}
$$


where $r \in[0,+\infty), \theta \in[0, \pi], \phi \sim \phi+2 \pi, \psi \sim \psi+4 \pi$. This amounts to regarding $\Phi_{1}, \Phi_{2}$ as coordinates on the total space of the tautological line bundle on $\mathbb{C P}^{1}$ parametrized by $\theta$ and $\phi$. The coordinates $r, \psi$ parametrize the fiber of this line bundle.

In the new coordinates the metric takes the form

$$
\mathrm{d} s^{2}=2 \mathrm{~d} r^{2}+\frac{r^{2}}{2}\left((\mathrm{~d} \psi-\cos \theta \mathrm{d} \phi)^{2}+\mathrm{d} \theta^{2}+\sin ^{2} \theta \mathrm{d} \phi^{2}\right)+k|\mathrm{~d} P|^{2} .
$$

The D-term constraint reads

$$
r^{2}+k \operatorname{Re} P=0
$$

It allows to eliminate (i.e. integrate out) Re $P$.

To eliminate $\operatorname{Im} P$, we gauge the $\mathrm{U}(1)$ isometry

$$
P \rightarrow P+i \alpha, \quad \psi \rightarrow \psi+2 \alpha,
$$

and then gauge away $\operatorname{Im} P$. Finally, we integrate out the gauge field.

The resulting four-dimensional metric has the form

$$
\mathrm{d} s^{2}=2 f(r) \mathrm{d} r^{2}+\frac{r^{2}}{2 f(r)}(\mathrm{d} \psi-\cos \theta \mathrm{d} \phi)^{2}+\frac{r^{2}}{2}\left(\mathrm{~d} \theta^{2}+\sin ^{2} \theta \mathrm{d} \phi^{2}\right)
$$

where

$$
f(r)=1+\frac{2 r^{2}}{k} .
$$

This metric is everywhere smooth and weakly curved for large $k$. By construction, it is guaranteed to be kählerian. The fermionic terms in the lagrangian are uniquely fixed by the requirement of $\mathcal{N}=2$ supersymmetry.

For general $n$ the result is similar. The target space is topologically a $\mathbb{C}^{n}$, but it is best regarded as the total space of the tautological line bundle on $\mathbb{C P}^{n-1}$. Let $A$ be the connection 1-form of the natural connection on this line bundle. (Its curvature is the standard Kähler form on $\left.\mathbb{C P}^{n-1}\right)$. Then the metric has the form

$$
\mathrm{d} s^{2}=2 \mathrm{~d} r^{2} f(r)+\frac{2 r^{2}}{n^{2} f(r)}(\mathrm{d} \psi-n A)^{2}+2 r^{2} d s_{F S}^{2} .
$$

Here the function $f(r)$ is the same as above, $d s_{F S}^{2}$ is the Fubini-Study metric on $\mathbb{C P}^{n-1}$, and $A$ is a connection 1 -form on $\mathbb{C P}^{n-1}$ whose curvature is the Fubini-Study Kähler form. The variable $\psi$ has period $2 \pi n$. On the affine chart $\mathbb{C}^{n-1} \subset \mathbb{C P}^{n-1}$ the metric $d s_{F S}^{2}$ and the 1 -form $A$ are given by

$$
\begin{aligned}
d s_{F S}^{2} & =\left(1+\sum_{k=1}^{n-1}\left|z_{k}\right|^{2}\right)^{-1} \sum_{i, j=1}^{n-1}\left(\delta_{i j}-\frac{z_{i} \bar{z}_{j}}{1+\sum_{k}\left|z_{k}\right|^{2}}\right) d \bar{z}_{i} d z_{j}, \\
A & =\frac{-i}{2} \frac{1}{1+\sum_{k}\left|z_{k}\right|^{2}} \sum_{i=1}^{n-1}\left(z_{i} d \bar{z}_{i}-\bar{z}_{i} d z_{i}\right) .
\end{aligned}
$$

Since the original LSM has $\mathrm{U}(n)$ flavor symmetry, the metric of the non-linear sigma model must have a $\mathrm{U}(n)$ isometry. This isometry is manifest in the above expression. 
Step 2. Now we need to find a solution to the one-loop beta-function equations which is kählerian, has a $\mathrm{U}(n)$ isometry, and asymptotes to the metric found in Step 1. The technology for doing this has been developed in ref. [16. After correcting some minor inaccuracies in ref. [16], we find that in the case $n=2$ there is a unique metric satisfying all these requirements:

$$
\mathrm{d} s^{2}=\frac{g_{2}(Y)}{2} \mathrm{~d} Y^{2}+\frac{1}{2 g_{2}(Y)}(\mathrm{d} \psi-\cos \theta \mathrm{d} \phi)^{2}+\frac{Y}{2}\left(\mathrm{~d} \theta^{2}+\sin ^{2} \theta \mathrm{d} \phi^{2}\right),
$$

where

$$
g_{2}(Y)=\frac{8}{k^{2}} \frac{Y}{e^{-4 Y / k}-1+\frac{4 Y}{k}} .
$$

The corresponding dilaton is given by

$$
\Phi=-\frac{2 Y}{k}+\text { const. }
$$

The variable $Y$ runs from 0 to $+\infty$, the variable $\psi$ has period $4 \pi$, while $\theta$ and $\phi$ are the usual coordinates on a unit 2-sphere.

The central charge can be found from

$$
c=6+3\left(2(\nabla \Phi)^{2}-\nabla^{2} \Phi\right),
$$

and comes out to be

$$
6\left(1+\frac{4}{k}\right)
$$

This agrees with eq. (3.2) and therefore provides a check on our computation.

The metric eq. (3.8) is everywhere smooth and weakly curved for large $k$. It agrees with eq. (3.8) in the region of large $Y$ (the corresponding change of variables is $Y=r^{2}$ ).

For general $n$ the result is similar. The metric and the dilaton are given by

$$
\begin{aligned}
\mathrm{d} s^{2} & =\frac{g_{n}(Y)}{2} \mathrm{~d} Y^{2}+\frac{2}{n^{2} g_{n}(Y)}(\mathrm{d} \psi-n A)^{2}+2 Y \mathrm{~d} s_{F S}^{2} \\
\Phi & =-\frac{n Y}{k},
\end{aligned}
$$

where the function $g_{n}(Y)$ is given by

$$
g_{n}(Y)=\frac{Y^{n-1}}{n} \frac{e^{2 n Y / k}}{\int_{0}^{Y} t^{n-1} e^{2 n t / k} d t} .
$$

The range of the variable $Y$ is the same as before, while $\psi$ has period $2 \pi n$. This is a unique kählerian metric which is everywhere smooth, has a $\mathrm{U}(n)$ isometry, solves the beta-function equations of motion, and asymptotes to the metric (3.5) for $Y \rightarrow \infty$. The central charge of this supergravity solution can be computed from

$$
c=3 n+3\left(2(\nabla \Phi)^{2}-\nabla^{2} \Phi\right) .
$$

It can be easily checked that it agrees with eq. (3.2). 


\section{T-Dual background and wrapped branes}

First, let us orbifold the background eq. (3.10) by the $\mathbb{Z}_{n}$ symmetry which acts on the target space coordinates by $\psi \rightarrow \psi+2 \pi$. The action on the fermions is fixed by the requirement that this symmetry commute with world-sheet supersymmetry. On the level of the LSM, we gauge the following discrete symmetry:

$$
\Phi_{i} \rightarrow \exp \left(\frac{2 \pi i}{n}\right) \Phi_{i}, \quad i=1, \ldots, n .
$$

Second, we set $n=2$ and tensor the above dilatonic background with an $\mathcal{N}=2$ minimal model [21]. In the supergravity approximation the latter can be thought of as the sigma model whose target space is the $\operatorname{disc}\left\{z=\mathrm{e}^{i \beta} \cos \rho\right\}$ with the metric

$$
\mathrm{d} s^{2}=m\left(d \rho^{2}+\cot ^{2} \rho d \beta^{2}\right), \quad m=2,3, \ldots,
$$

and the dilaton

$$
\Phi=-\log \sin \rho+\text { const }
$$

There is a genuine curvature singularity at the boundary of the disc, $\rho=0$, where the dilaton blows up. However, this is simply due to a bad choice of description and a T-duality makes it regular there [22]. This supergravity solution has an obvious U(1) isometry. But the corresponding CFT preserves only its $\mathbb{Z}_{m}$ subgroup. The breaking can be attributed to world-sheet instanton effects.

Finally, we take a $\mathbb{Z}_{m}$ orbifold of the product CFT to eliminate fractional R-charges. The relevant orbifold group is generated by the combination of $\psi \rightarrow \psi+2 \pi / m$ and the generator of the $\mathbb{Z}_{m}$ symmetry of the minimal model. Since we have taken the $\mathbb{Z}_{2}$ orbifold at the first step, the total orbifold group is $\mathbb{Z}_{2 m}$. As usual, the orbifold theory has a quantum $\mathbb{Z}_{2 m}$ symmetry, which we denote $\mathbb{Z}_{2 m}^{\text {qua }}$.

The central charge of the minimal model is

$$
c_{\min }=3\left(1-\frac{2}{m}\right), \quad m=2,3, \ldots
$$

Thus if we set $k=4 m$, the total central charge is

$$
c_{\mathrm{tot}}=3\left(1-\frac{2}{m}\right)+6\left(1+\frac{1}{m}\right)=9 .
$$

Hence we can obtain a bona fide superstring background with $c=15$ by throwing in two more free chiral superfields.

The final result is an $\mathcal{N}=2 \mathrm{CFT}$ which describes a type-II superstring background with four-dimensional Poincaré invariance and an additional $\mathrm{U}(2)$ symmetry. $\mathcal{N}=2$ superconformal symmetry on the world-sheet gives rise to $\mathcal{N}=2 d=4$ supersymmetry in target space in the usual manner. The quantum symmetry $\mathbb{Z}_{2 m}^{\text {qua }}$ on the worlsheet corresponds to a $\mathbb{Z}_{2 m}$ R-symmetry in target space, as we will see momentarily. 
To establish a connection with NS five-branes wrapped on $\mathbb{C P}^{1}$, we perform a T-duality along a Killing vector field

$$
v_{K}=\frac{1}{m}\left(\frac{\partial}{\partial \beta}+\frac{\partial}{\partial \psi}\right)
$$

Note that this vector field generates a circle action of period $2 \pi$ because of the $\mathbb{Z}_{m}$ orbifold on the last step. Thus, the dual variable, which we denote by $\lambda$, will also have period $2 \pi$. The T-dual background has the following form:

$$
\begin{aligned}
\mathrm{d} s^{2}= & \frac{g_{2}(Y)}{2} \mathrm{~d} Y^{2}+\frac{Y}{2}\left(\mathrm{~d} \theta^{2}+\sin ^{2} \theta \mathrm{d} \phi^{2}\right)+m \mathrm{~d} \rho^{2}+\left(1+2 m \cot ^{2} \rho g_{2}(Y)\right)^{-1} \times \\
& \times\left(m \cot ^{2} \rho(\mathrm{d} \psi-\cos \theta \mathrm{d} \phi)^{2}+2 m^{2} g_{2}(Y) \mathrm{d} \lambda^{2}\right), \\
B= & \frac{m}{2 \pi} \frac{\mathrm{d} \lambda \wedge(\mathrm{d} \psi-\cos \theta \mathrm{d} \phi)}{1+2 m \cot ^{2} \rho g_{2}(Y)} \\
\Phi= & -\frac{Y}{2 m}-\frac{1}{2} \log \left(\frac{m}{2} \cos ^{2} \rho+\frac{\sin ^{2} \rho}{4 g_{2}(Y)}\right)+\text { const. }
\end{aligned}
$$

The function $g_{2}(Y)$ is given by

$$
g_{2}(Y)=\frac{1}{2 m} \frac{Y / m}{e^{-Y / m}-1+\frac{Y}{m}} .
$$

The T-dual background has a curvature singularity at the locus

$$
Y=0, \quad \rho=\frac{\pi}{2} .
$$

The dilaton and the norm of H-field also diverge there. At large $Y$, the function $g_{2}(Y)$ approaches $1 / 2 m$, and the solution asymptotes to $\Phi \sim-Y / 2 m$ and

$$
\begin{aligned}
\mathrm{d} s^{2} \sim & \frac{1}{4 m} \mathrm{~d} Y^{2}+\frac{Y}{2}\left(\mathrm{~d} \theta^{2}+\sin ^{2} \theta \mathrm{d} \phi^{2}\right)+ \\
& +m\left[\mathrm{~d} \rho^{2}+\cos ^{2} \rho(\mathrm{d} \psi-\cos \theta \mathrm{d} \phi)^{2}+\sin ^{2} \rho \mathrm{d} \lambda^{2}\right], \\
B \sim & \frac{m}{2 \pi} \sin ^{2} \rho \mathrm{d} \lambda \wedge(\mathrm{d} \psi-\cos \theta \mathrm{d} \phi) .
\end{aligned}
$$

For a fixed value of $(\theta, \phi)$, the metric for $(\rho, \psi, \lambda)$ is that of a round $\mathbb{S}^{3}$ realized by $\left|z_{1}\right|^{2}+$ $\left|z_{2}\right|^{2}=m$, where $z_{1}=\sqrt{m} \mathrm{e}^{i \lambda} \sin \rho$ and $z_{2}=\sqrt{m} \mathrm{e}^{i \psi} \cos \rho$. We also note that the $H$ flux through any such $\mathbb{S}^{3}$ is $2 \pi m$. Thus we have an $\mathrm{SU}(2)$ WZW model fibered over $\mathbb{C P}^{1}$, where the fibration is determined by the combination $(\mathrm{d} \psi-\cos \theta \mathrm{d} \phi)$ that appears in the solution. Note that $A=\cos \theta \mathrm{d} \phi$ is the Levi-Civita connection of $\mathbb{C P}^{1}$ (and $\frac{1}{2} A$ is the connection on the spin bundle $\left.\operatorname{Spin}\left(\mathbb{C P}^{1}\right)\right)$. Note also that the shift $\psi \rightarrow \psi-2 \gamma$ corresponds to the $\mathrm{U}(1)_{\text {twist }}$ action (2.3) for the $\mathbb{S}^{3}$ coordinates $g=z_{1}+j z_{2}$. that is used in the $\mathcal{N}=2$ twisting. Thus at large $Y$ the geometry is that of an $\mathrm{SU}(2)$ bundle over $\mathbb{C P}^{1}$ associated with a circle bundle with unit Euler number via the action of $\mathrm{U}(1)_{\text {twist }}$. This shows that we are dealing with the $\mathcal{N}=2$ compactification of $m$ NS5-branes on $\mathbb{C P}^{1}$.

The $\mathrm{U}(1)_{R}$ action (2.2) is $z_{1}+j z_{2} \rightarrow \mathrm{e}^{i \alpha}\left(z_{1}+j z_{2}\right) \mathrm{e}^{i \alpha}$ and hence is a shift $\lambda \rightarrow \lambda+2 \alpha$. It measures twice the momentum in $\lambda$-direction. In the original description (before $\mathrm{T}$ duality), it measures twice the winding in the direction $\mathrm{U}(1)_{K}$ given by the Killing vector 
field $v_{K}$ in (4.1). However, a string which is wound $2 m$ times around $\mathrm{U}(1)_{K}$ lifts to a well-defined configuration in the geometry before the $\mathbb{Z}_{2 m}$ orbifold. Such a configuration is topologically trivial since the total geometry before orbifolding has no non-contracable loop. Thus, the winding number in $\mathrm{U}(1)_{K}$ is only conserved modulo $2 m$. (The conserved charge is that of the quantum symmetry $\mathbb{Z}_{2 m}^{\text {qua }}$.) In other words, momentum in $\lambda$-direction is conserved only modulo $2 m$. This corresponds to the anomaly of the $\mathrm{U}(1)_{R}$ symmetry

$$
\mathrm{U}(1)_{R} \rightarrow \mathbb{Z}_{4 m}
$$

We have shown, as promised, that (the $\mathbb{Z}_{2}$-quotient of) this R-symmetry is nothing but the quantum symmetry $\mathbb{Z}_{2 m}^{\text {qua }}$. On the other hand, the $\mathrm{U}(1)$ subgroup of the $\mathrm{SU}(2)_{R}$ shifts $\psi$, and remains as a symmetry of the system.

In refs. [12, 13] a one-parameter family of supergravity solutions has been found which describes five-branes wrapped on $\mathbb{C P}^{1}$. The parameter, denoted in ref. [12] by $k$, can take any real value. For $k<-1$ the solutions have a naked singularity of a "bad" kind according to the criteria of ref. [23], while for $k \geq 1$ the naked singularity, although still present, is of a "good" kind. It is easy to check that our solution coincides with the solution of refs. [12, 13 , in the special case $k=-1$. The above-mentioned singularity is at $Y=0, \rho=\frac{\pi}{2}$. However, now it is clear that the singularity is an artefact of the supergravity approximation. The world-sheet CFT is manifestly non-singular.

The authors of ref. [12] proposed that the solution with $k=-1$ represents the origin of the Coulomb branch of the five-brane theory, while the solutions with $k>-1$ correspond to a one-dimensional submanifold of the Coulomb branch. It is also proposed that the solutions with $k<-1$ are unphysical. In section 5 we use a more general LSM to derive the supergravity solution for general $k \geq-1$. Our analysis confirms the above interpretation of $k$, with some important clarifications.

Note that we have a well-defined world-sheet CFT only for $m>1$. This is a signal that there is no decoupled theory on a single five-brane [24, 25.

Similarly, we can show that the six-dimensional linear dilaton background corresponding to $n=3$ is related to NS five-branes wrapped on $\mathbb{C P}^{2}$. To this end we set $k=9 m, m=$ $2,3, \ldots$, tensor with the $m^{\text {th }} \mathcal{N}=2$ minimal model, and then take the $\mathbb{Z}_{m}$ orbifold. The resulting $(2,2)$ superconformal field theory has central charge $c=12$. Therefore we can obtain a $(2,2)$ superstring background by tensoring with a single free chiral superfield. To establish a connection with wrapped five-branes, we perform T-duality along the Killing vector field

$$
\frac{1}{m}\left(\frac{\partial}{\partial \beta}+\frac{\partial}{\partial \psi}\right)
$$

The resulting eight-dimensional metric, B-field, and the dilaton have the following form:

$$
\begin{aligned}
\mathrm{d} s^{2}= & \frac{g_{3}(Y)}{2} d Y^{2}+2 Y \mathrm{~d} s_{F S}^{2}+m \mathrm{~d} \rho^{2}+\left(1+\frac{9 m}{2} \cot ^{2} \rho g_{3}(Y)\right)^{-1} \times \\
& \times\left(m \cot ^{2} \rho(\mathrm{d} \psi-3 A)^{2}+\frac{9}{2} m^{2} g_{3}(Y) \mathrm{d} \lambda^{2}\right)
\end{aligned}
$$




$$
\begin{aligned}
B & =\frac{m}{2 \pi} \frac{\mathrm{d} \lambda \wedge(\mathrm{d} \psi-3 A)}{1+\frac{9 m}{2} \cot ^{2} \rho g_{3}(Y)} \\
\Phi & =-\frac{Y}{3 m}-\frac{1}{2} \log \left(\frac{9 m}{2} \cos ^{2} \rho+\frac{\sin ^{2} \rho}{g_{3}(Y)}\right)+\text { const. }
\end{aligned}
$$

Here the variables $\psi$ and $\lambda$ have period $2 \pi$, and the function $g_{3}(Y)$ is given by

$$
g_{3}(Y)=\frac{2}{9 m} \frac{\frac{1}{2}\left(\frac{2 Y}{3 m}\right)^{2}}{1-\frac{2 Y}{3 m}+\frac{1}{2}\left(\frac{2 Y}{3 m}\right)^{2}-e^{-2 Y /(3 m)}} .
$$

At large $Y$, the background is an $\mathbb{S}^{3}$ with H-flux fibered over $\mathbb{C P}^{2}$. The fibration is associated with the $\mathrm{U}(2)$ frame bundle of $\mathbb{C P}^{2}$ and the $\mathrm{U}(2)$ action on $\mathbb{S}^{3}$ determined by the twist we have chosen in section 2. Thus, we are dealing with a $\mathbb{C P}^{2}$ compactification of NS5-branes with $(2,2)$ supersymmetry in $1+1$ dimensions. The vector U(1) R-symmetry is associated with the action (2.3) and shifts $\psi$. This remains a symmetry of the system. On the other hand, the axial U(1) R-symmetry, identified as the action (2.2), shifts $\lambda$ and is broken to $\mathbb{Z}_{6 m}$. Since in $(2,2)$ super-Yang-Mills the axial U(1) R-symmetry is anomaly free, this is somewhat puzzling. A possible resolution will be discussed in section 8 .

This solution has a curvature singularity at $Y=0, \rho=\pi / 2$. There is also a $\mathbb{C}^{3} / \mathbb{Z}_{3}$ orbifold singularity at $Y=0, \rho=0$. This is related to the fact that the solution describes five-branes wrapped on the exceptional divisor of the crepant resolution of a $\mathbb{C}^{3} / \mathbb{Z}_{3}$ singularity. The Kähler class of the exceptional divisor is zero for the above solution. A more general solution allowing a non-zero size for the exceptional divisor is written down in section 7 .

One can also interpret the $n=4 \mathrm{LSM}$ with $k=16 m$ tensored with the $m^{\text {th }} \mathcal{N}=2$ minimal model as representing $m$ euclidean NS five-branes wrapped on $\mathbb{C P}^{3}$ in a Calabi-Yau 4-fold. We will not discuss this case any further in this paper.

\section{Mirror description of wrapped five-branes}

Let us recapitulate what we have seen. We denote by $\operatorname{LSM}_{k}^{(n)}$ the IR fixed point of the linear sigma model (3.1). We first focus on the case $n=2$ which we denote simply by $\mathrm{LSM}_{k}$. We considered the $\mathcal{N}=2 \mathrm{SCFT}$

$$
\mathcal{C}_{m}=\left[\frac{\mathrm{LSM}_{4 m}}{\mathbb{Z}_{2}} \times \frac{\mathrm{SU}(2)_{m-2}}{\mathrm{U}(1)}\right] / \mathbb{Z}_{m}^{\text {diag }},
$$

where $\frac{\mathrm{SU}(2)_{m-2}}{\mathrm{U}(1)}$ stands for the level $(m-2)$ minimal model, $\mathbb{Z}_{2}$ corresponds to the shift of $\operatorname{Im} P$ by half-period, and $\mathbb{Z}_{m}^{\text {diag }}$ is the diagonal subgroup of the $\mathrm{U}(1) \times \mathbb{Z}_{m}$ symmetry of the product theory. The model $\mathrm{LSM}_{4 m}$ has an asymptotic region which is described by $\mathbb{C P}^{1} \times \mathbb{R} \times \mathrm{U}(1)_{4 m}$ where $\mathbb{R}$ is the linear dilaton and $\mathrm{U}(1)_{4 m}$ is the circle of radius $\sqrt{4 m}$. The $\mathbb{Z}_{2}$ orbifold group acts only on the $\mathrm{U}(1)_{4 m}$ factor, resulting in $\mathrm{U}(1)_{4 m} / \mathbb{Z}_{2}=\mathrm{U}(1)_{m}$. Then using T-duality $\left[\mathrm{U}(1)_{m} \times \frac{\mathrm{SU}(2)_{m-2}}{\mathrm{U}(1)}\right] / \mathbb{Z}_{m} \cong \mathrm{SU}(2)_{m-2}$ [6, 21], we find that the asymptotic region describes $m$ NS5-branes wrapped on $\mathbb{C P}^{1}$. The theory (5.1) can also be regarded as a $\mathbb{Z}_{2 m}$ orbifold of LSM $\times \frac{\mathrm{SU}(2)}{\mathrm{U}(1)}$, and has the associated quantum symmetry $\mathbb{Z}_{2 m}^{\text {qua }}$. 
To find the mirror description of $\mathcal{C}_{m}$, we use the standard fact about orbifolds: the orbfold of a CFT $\mathcal{C}$ by some discrete symmetry $G$ has a quantum symmetry $G^{\prime}$ isomorphic to $G$, such that its orbifold reproduces the original CFT, $(\mathcal{C} / G) / G^{\prime} \cong \mathcal{C}$. Applying this to $\mathcal{C}_{m}$ in (5.1) and $G=\left(\mathbb{Z}_{m} \times \mathbb{Z}_{m}\right) / \mathbb{Z}_{m}^{\text {diag }} \cong \mathbb{Z}_{m}$, we find that $\mathcal{C}_{m} / G=\frac{\mathrm{LSM}_{4 m}}{\mathbb{Z}_{2}} / \mathbb{Z}_{m} \times \frac{\mathrm{SU}(2)_{m-2}}{\mathrm{U}(1)} / \mathbb{Z}_{m}$, and therefore

$$
\mathcal{C}_{m} \cong\left[\frac{\mathrm{LSM}_{4 m}}{\mathbb{Z}_{2 m}} \times \frac{\mathrm{SU}(2)_{m-2}}{\mathrm{U}(1)} / \mathbb{Z}_{m}\right] / \mathbb{Z}_{m}^{\prime}
$$

where we have used $\frac{\mathrm{LSM}_{4 m}}{\mathbb{Z}_{2}} / \mathbb{Z}_{m} \cong \mathrm{LSM}_{4 m} / \mathbb{Z}_{2 m}$.

It is known that the orbifold $\frac{\mathrm{SU}(2)_{m-2}}{\mathrm{U}(1)} / \mathbb{Z}_{m}$ is mirror to the original minimal model, which can be realized as the IR limit of the LG model of a single chiral superfield $X$ with the superpotential $W=X^{m}$. The model $\operatorname{LSM}_{4 m} / \mathbb{Z}_{2 m}$ also has a mirror description, as shown in ref. [17]. It is given by two chiral superfields $Y$ and $Z$ of periodicity $2 \pi i$, with the following Kahler potential and superpotential

$$
\begin{aligned}
& K=\frac{m}{2}|Z|^{2}+a|Y|^{2}, \\
& W=\mathrm{e}^{-m Z}\left(\mathrm{e}^{-Y}+\mathrm{e}^{Y}\right) .
\end{aligned}
$$

The parameter $a$ is mirror to the squashing of the metric of $\mathbb{C P}^{1}$, with the round metric corresponding to the degenerate limit $a \rightarrow 0$. This mirror Landau-Ginzburg theory can be obtained as follows. To incorporate the orbifold action, we must change the $P$-dependent part of the action (3.1) from $\frac{k}{4}(P+\bar{P}+V)^{2}$ to $\frac{1}{4 m}(P+\bar{P}+2 m V)^{2}$, where $P$ transforms as $P \rightarrow P+2$ mia under the gauge transformation. The period of $\operatorname{Im} P$ is still $2 \pi$. Applying the method of ref. [18], we find that the mirror is a LG model of three chiral superfields $Y_{1}, Y_{2}, Y_{P}$, all with period $2 \pi i$, which are constrained by $Y_{1}+Y_{2}+2 m Y_{P}=0$. The superpotential is given by $W=\mathrm{e}^{-Y_{1}}+\mathrm{e}^{-Y_{2}}$. The Kähler potential is degenerate for a round $\mathbb{C P}^{1}$ metric, $K=\frac{m}{2}\left|Y_{P}\right|^{2}$, but can be made into a regular one $K=\frac{m}{2}\left|Y_{P}\right|^{2}+a\left|\frac{Y_{1}-Y_{2}}{2}\right|^{2}$ by squashing $\mathbb{C P}^{1}$. We solve the constraint by setting $Y_{1}=Y+m Z, Y_{2}=-Y+m Z$, $Y_{P}=-Z$, and obtain the above Kähler potential and superpotential.

Thus, the mirror of the product theory $\frac{\mathrm{LSM}_{4 m}}{\mathbb{Z}_{2 m}} \times \frac{\mathrm{SU}(2)_{m-2}}{\mathrm{U}(1)} / \mathbb{Z}_{m}$ is a LG model with the superpotential

$$
W=\mathrm{e}^{-m Z}\left(\mathrm{e}^{-Y}+\mathrm{e}^{Y}\right)+X^{m} .
$$

The theory $\mathcal{C}_{m}$ is therefore mirror to the orbifold of this LG model with respect to the $\mathbb{Z}_{m}^{\prime}$ discrete symmetry. The orbifold group $\mathbb{Z}_{m}^{\prime}$ acts on the fields as follows:

$$
\mathbb{Z}_{m}^{\prime}: Y \rightarrow Y, \quad Z \rightarrow Z-\frac{2 \pi i}{m}, \quad X \rightarrow \mathrm{e}^{\frac{2 \pi i}{m}} X
$$

The action of the quantum symmetry $\mathbb{Z}_{2 m}^{\text {qua }}$ is $Y \rightarrow Y+\pi i, Z \rightarrow Z+\frac{\pi i}{m}, X \rightarrow X$. We also note that the LG model has a dilaton represented by the linear term in the supercurrent $\left.\mathcal{J}\right|_{\text {linear }}=\left(\partial_{0}-\partial_{1}\right) \operatorname{Im}(Z)$. Thus the dilaton is exactly linear:

$$
\Phi=-\operatorname{Re} Z
$$

We see that the string coupling is weak in the asymptotic region $\operatorname{Re} Z \gg 0$, while the strong coupling region $\operatorname{Re} Z \ll 0$ is effectively blocked by the Liouville-type interaction. 
Conformal field theories based on the SU(2) current algebra are classified by simply laced Lie groups

$$
\Gamma_{m}= \begin{cases}A_{m-1} & (m \text { integer } \geq 2), \\ D_{\frac{m}{2}+1} & (m \text { even integer } \geq 6), \\ E_{6} & (m=12), \\ E_{7} & (m=18), \\ E_{8} & (m=30),\end{cases}
$$

where $(m-2)$ is the level of the current algebra. They have central charge $c=3(m-2) / m$ and are denoted by $\mathrm{SU}(2)_{\Gamma_{m}}$. $\mathrm{SU}(2)_{A_{m-1}}$ is the one we have been calling $\mathrm{SU}(2)_{m-2}$ and corresponds to the system of $m$ ordinary NS5-branes. Others corresponds to other types of NS5-branes. For all $\Gamma_{m}$ we have the $\mathcal{N}=2$ supercoset $\mathrm{SU}(2)_{\Gamma_{m}} / \mathrm{U}(1)$ which is called the $\mathcal{N}=2$ minimal model of type $\Gamma_{m}$. These models arise as the IR fixed point of the LG models with the following superpotentials [26, 27]:

$$
\begin{aligned}
W_{A_{m-1}} & =X_{1}^{m}+X_{2} X_{3}, \\
W_{D_{\frac{m}{2}+1}} & =X_{1}^{\frac{m}{2}}+X_{1} X_{2}^{2}+X_{3}^{2}, \\
W_{E_{6}} & =X_{1}^{4}+X_{2}^{3}+X_{3}^{2}, \\
W_{E_{7}} & =X_{1}^{3} X_{2}+X_{2}^{3}+X_{3}^{2}, \\
W_{E_{8}} & =X_{1}^{5}+X_{2}^{3}+X_{3}^{2} .
\end{aligned}
$$

They have discrete $\mathbb{Z}_{m}$ symmetry which acts on the LG fields by

$$
X_{i} \rightarrow \mathrm{e}^{2 \pi i q_{i}} X_{i}
$$

where $q_{i}$ is the R-charge of $X_{i}$ such that $W\left(\lambda^{q_{i}} X_{i}\right)=\lambda W\left(X_{i}\right)$. Note that we have

$$
q_{1}+q_{2}+q_{3}=\frac{m+1}{m}
$$

As in the $A_{m-1}$ model, we have an identity

$$
\left[\mathrm{U}(1)_{m} \times \frac{\mathrm{SU}(2)_{\Gamma_{m}}}{\mathrm{U}(1)}\right] / \mathbb{Z}_{m} \cong \mathrm{SU}(2)_{\Gamma_{m}}
$$

Then it follows that $\Gamma_{m}$-type NS5-brane wrapped on $\mathbb{C P}^{1}$ in Eguchi-Hanson space is described by the CFT given by (5.1) or (5.2), with $\mathrm{SU}(2)_{m-2}$ replaced by $\mathrm{SU}(2)_{\Gamma_{m}}$. Repeating the arguments leading to eq. (5.5), we find that the mirror is a LG orbifold with the superpotential

$$
W=\mathrm{e}^{-m Z}\left(\mathrm{e}^{-Y}+\mathrm{e}^{Y}\right)+W_{\Gamma_{m}}
$$

where the orbifold group $\mathbb{Z}_{m}^{\prime}$ is generated by $Y \rightarrow Y$ and

$$
Z \rightarrow Z-\frac{2 \pi i}{m}, \quad X_{i} \rightarrow \mathrm{e}^{2 \pi i q_{i}} X_{i}
$$


Similarly, the theory describing $\Gamma_{m}$-type NS5-brane wrapped on $\mathbb{C P}^{2}$ is given by

$$
\begin{aligned}
\mathcal{C}_{\Gamma_{m}}^{(3)} & =\left[\frac{\mathrm{LSM}_{9 m}^{(3)}}{\mathbb{Z}_{3}} \times \frac{\mathrm{SU}(2)_{\Gamma_{m}}}{\mathrm{U}(1)}\right] / \mathbb{Z}_{m}^{\text {diag }} \\
& \cong\left[\frac{\mathrm{LSM}_{9 m}^{(3)}}{\mathbb{Z}_{3 m}} \times \frac{\mathrm{SU}(2)_{\Gamma_{m}}}{\mathrm{U}(1)} / \mathbb{Z}_{m}\right] / \mathbb{Z}_{m}^{\prime} .
\end{aligned}
$$

The mirror is the LG model with the superpotential

$$
W=\mathrm{e}^{-m Z}\left(\mathrm{e}^{-Y_{1}}+\mathrm{e}^{-Y_{2}}+\mathrm{e}^{Y_{1}+Y_{2}}\right)+W_{\Gamma_{m}},
$$

divided by the orbifold group $\mathbb{Z}_{m}^{\prime}$ whose action on the fields is given by $Y_{i} \rightarrow Y_{i}$ and eq. (5.18). The theory has a quantum symmetry $\mathbb{Z}_{3 m}^{\text {qua }}$ and it acts on the mirror fields as $Y_{i} \rightarrow Y_{i}+\frac{2 \pi i}{3}, Z \rightarrow Z-\frac{2 \pi i}{3 m}, X_{i} \rightarrow X_{i}$.

\section{Non-perturbative $4 \mathrm{~d}$ physics from the world-sheet}

\subsection{Derivation of the Seiberg-Witten solution}

The LG orbifold (5.5)- (5.6) has a finite-dimensional chiral ring spanned by $\mathbb{Z}_{m}^{\prime}$-invariant operators

$$
\mathrm{e}^{-(m-\ell) Z} X^{\ell}, \quad \ell=0,1, \ldots,(m-2) .
$$

All these operators are exactly marginal. Let us deform the theory by adding these operators to $W$ :

$$
W=\mathrm{e}^{-m Z}\left(\mathrm{e}^{-Y}+\mathrm{e}^{Y}\right)+X^{m}+\sum_{\ell=0}^{m-2} u_{\ell} \mathrm{e}^{-(m-\ell) Z} X^{\ell} .
$$

Since the parameters $u_{\ell}$ are exactly marginal, eq. (6.2) represents a deformed string background. Note that the deformation breaks the quantum symmetry $\mathbb{Z}_{2 m}^{\text {qua }}$ to its subgroup. As in eq. (5.9), we can add two extra variables $X_{2}$ and $X_{3}$ with the superpotential $X_{2} X_{3}$ without changing the IR fixed point.

Let us change the variables by setting $X=\mathrm{e}^{-Z} \tilde{X}, X_{2}=\mathrm{e}^{-m Z} \widetilde{X}_{2}$ and $X_{3}=\widetilde{X}_{3}$, where the tilded variables are all invariant under $\mathbb{Z}_{m}^{\prime}$. Then $\mathrm{e}^{-m Z}$ factors out in the deformed superpotential:

$$
W=\mathrm{e}^{-m Z}\left(\mathrm{e}^{-Y}+\mathrm{e}^{Y}+\widetilde{X}^{m}+\sum_{\ell=0}^{m-2} u_{\ell} \widetilde{X}^{\ell}+\widetilde{X}_{2} \widetilde{X}_{3}\right)
$$

We now show that this LG model is weakly equivalent (in the sense of [18]) to the non-linear sigma model on the non-compact Calabi-Yau 3-fold

$$
\mathrm{e}^{-Y}+\mathrm{e}^{Y}+\widetilde{X}^{m}+\sum_{\ell=0}^{m-2} u_{\ell} \widetilde{X}^{\ell}+\widetilde{X}_{2} \widetilde{X}_{3}=0 .
$$


Weak equivalence means that central charges of A-branes in the two theories agree. The central charge of an A-brane in the LG model is measured by the period integral [18, 28]

$$
\Pi=\int \frac{1}{g_{s}} \mathrm{~d} Y \mathrm{~d} Z \mathrm{~d} X \mathrm{~d} X_{2} \mathrm{~d} X_{3} \exp (-W),
$$

where $g_{s}$ is the string coupling, and the integration is over a lagrangian 5-cycle wrapped by the A-brane. This formula was derived assuming that the dilaton is constant, but in the present theory the dilaton is given by eq. (5.7), and thus the string coupling is $g_{s}=\mathrm{e}^{\Phi_{\text {dilaton }}}=\mathrm{e}^{-\operatorname{Re} Z}$. It is easy to see how the expression (6.5) should be modified so that it is holomorphic in all variables: one should simply replace $g_{s}$ with $e^{-Z}$. Then the period integral eq. (6.5) becomes

$$
\Pi=\int \mathrm{e}^{Z} \cdot \mathrm{d} Y \mathrm{~d} Z \mathrm{e}^{-Z} \mathrm{~d} \widetilde{X} \mathrm{e}^{-m Z} \mathrm{~d} \widetilde{X}_{2} \mathrm{~d} \widetilde{X}_{3} \exp (-W) .
$$

We see that the jacobian $\mathrm{e}^{-Z}$ from the change of variables $X=\mathrm{e}^{-Z} \tilde{X}$ is cancelled by the complexified string coupling. This enables us to perform the $\mathrm{e}^{-m Z}$-integration which yields

$$
\Pi=\int \mathrm{d} Y \mathrm{~d} \widetilde{X} \mathrm{~d} \widetilde{X}_{2} \mathrm{~d} \widetilde{X}_{3} \delta\left(\mathrm{e}^{-Y}+\mathrm{e}^{Y}+\widetilde{X}^{m}+\sum_{\ell=0}^{m-2} u_{\ell} \widetilde{X}^{\ell}+\widetilde{X}_{2} \widetilde{X}_{3}\right) .
$$

This is none other but the period integral of the Calabi-Yau space (6.4), which measures the central charge of an A-brane. It is well known [29, 30] that the type-IIB superstring on this space reduces in the low energy limit to the Seiberg-Witten theory of the curve

$$
\mathrm{e}^{-Y}+\mathrm{e}^{Y}+\widetilde{X}^{m}+\sum_{\ell=0}^{m-2} u_{\ell} \widetilde{X}^{\ell}=0
$$

with the meromorphic differential $\lambda=\widetilde{X} \mathrm{~d} Y$. This is exactly the low energy effective theory of the $\mathrm{SU}(m)$ super-Yang-Mills, where $u_{\ell}$ 's represent the Coulomb branch vevs. The breaking of the quantum symmetry $\mathbb{Z}_{2 m}^{\text {qua }}$ corresponds to the spontaneous breaking of the $\mathbb{Z}_{4 m}$ R-symmetry.

This can be easily generalized to all ADE fivebranes. The change of variables is $X_{i}=\mathrm{e}^{-m q_{i} Z} \widetilde{X}_{i}$, and the holomorphic measure is given by

$$
\frac{1}{g_{s}} \mathrm{~d} Y \mathrm{~d} Z \prod_{i=1}^{3} \mathrm{~d} X_{i}=\mathrm{d} Y \mathrm{e}^{-m Z} \mathrm{~d} Z \prod_{i=1}^{3} \mathrm{~d} \widetilde{X}_{i}
$$

where we used $\sum_{i=1}^{3} q_{i}=(m+1) / m$ and $g_{s}=\mathrm{e}^{-Z}$. It follows that the deformed system is weakly equivalent to the sigma model on the Calabi-Yau 3-fold

$$
\mathrm{e}^{-Y}+\mathrm{e}^{Y}+W_{\Gamma_{m}}\left(\widetilde{X}_{i}, u_{\ell}\right)=0
$$

where $W_{\Gamma_{m}}\left(X_{i}, u_{\ell}\right)$ is a versal deformation of $W_{\Gamma_{m}}\left(X_{i}\right)$. This is exactly the Calabi-Yau geometry that reproduces the Seiberg-Witten effective theory of $\mathcal{N}=2$ super-Yang-Mills 
with gauge group $\Gamma_{m}$ [29]. The connection between the five-brane theory and $\mathcal{N}=2$ super-Yang-Mills is discussed in more detail in section 8.

Given the equivalence of the decoupled theory of type-IIB NS5-branes and that of the ADE singularity in type-IIA, the present system is equivalent to type-IIA strings on a non-compact Calabi-Yau manifold with an ADE singularity along $\mathbb{C P}^{1}$. In fact such systems are studied in the so called "geometric engineering" approach [30], in which the Seiberg-Witten solution was also reproduced using local mirror symmetry. We also note that a transform from a similar LG model to the CY geometry was performed in [31].

\subsection{Argyres-Douglas points}

It is not easy to trace back what a generic deformation of $W$ does to the wrapped five-brane system. However, there is a particular family which has a simple but interesting meaning. Consider the following one-parameter deformation that breaks $\mathbb{Z}_{2 m}^{\text {qua }}$ to $\mathbb{Z}_{m}$ :

$$
W=\mathrm{e}^{-m Z}\left(\mathrm{e}^{-Y}+\mathrm{e}^{Y}\right)+X^{m}+\mathrm{e}^{t / 2} \mathrm{e}^{-m Z} .
$$

That is, we set all $u_{\ell}$ to zero except $u_{0}=\mathrm{e}^{t / 2}$. By reversing the dualization process, we find that this deformation corresponds to replacing $\mathrm{LSM}_{4 m} / \mathbb{Z}_{2}$ in (5.1) by the IR fixed point of the following linear sigma model. The gauge group is $\mathrm{U}(1) \times \mathrm{U}(1)$, and there are four

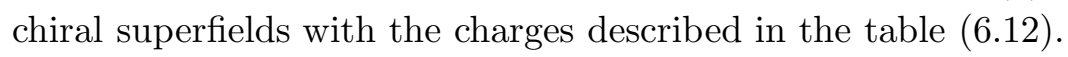

$$
\begin{array}{ccccc} 
& \Phi_{1} & \Phi_{2} & Q & P \\
\mathrm{U}(1) & 1 & 1 & 0 & \text { shift } \\
\mathrm{U}(1) & 1 & 1 & -2 & 0
\end{array}
$$

We have added one field $Q$ and one $\mathrm{U}(1)$ gauge group to the basic linear sigma model for $\mathrm{LSM}_{4 m}$. The orbifold group $\mathbb{Z}_{m}^{\text {diag }}$ acts on the minimal model as before and on the LSM as $\Phi_{i} \rightarrow \Phi_{i}, Q \rightarrow \mathrm{e}^{\frac{2 \pi i}{m}} Q$ and $P \rightarrow P$. The breaking $\mathbb{Z}_{2 m}^{\text {qua }} \rightarrow \mathbb{Z}_{m}$ corresponds to the fact that the final system can only be regarded as a $\mathbb{Z}_{m}$ orbifold.

The new U(1) has a non-trivial Fayet-Iliopoulos-theta parameter which is equal to $t$. One can easily see that the complex geometry of the Higgs branch is that of a resolved $A_{1}$ singularity, or Eguchi-Hanson space, where $t$ is roughly (up to world-sheet instanton corrections) the size of $\mathbb{C P}^{1}$. This is discussed in greater detail below. The original undeformed CFT - the Landau-Ginzburg without the $\mathrm{e}^{t / 2} \mathrm{e}^{-m Z}$-term - corresponds to $t \rightarrow-\infty$, the orbifold limit of the Eguchi-Hanson space. The other limit $t \rightarrow+\infty$ is the large volume limit. Formally, this can be seen as follows. Making a change of variables $Z=Z^{\prime}+t /(2 m)$ and taking the strict $t \rightarrow+\infty$ limit, we find the superpotential

$$
W=\mathrm{e}^{-m Z^{\prime}}+X^{m} .
$$

This is indeed the mirror dual of $m$ flat NS5-branes [8, 9].

For generic $t$ the infrared limit of this LSM is a non-singular CFT, which can be thought of as a non-linear sigma-model of the Higgs branch obtained by integrating out the vector multiplets. But for special values of $t$ it may happen that the effective twisted superpotential for the field-strength superfield $\Sigma=\bar{D}_{+} D_{-} V$ vanishes; for these values of 
$t$, a "Coulomb branch" develops and the CFT becomes singular [32. In our model the effective twisted superpotential is ${ }^{3}$

$$
W_{\text {eff }}=-t \Sigma-\sum_{i=1}^{3} Q_{i} \Sigma\left(\log \left(Q_{i} \Sigma\right)-1\right)=(-t+2 \log (-2)) \Sigma,
$$

where $Q_{i}$ stands for the charge vector $(1,1,-2)$. We see that the system is singular exactly at one point

$$
t=\log 4
$$

Let us see what happens to the mirror theory at this point. Since $\mathrm{e}^{t / 2}=\sqrt{\mathrm{e}^{\log 4}}= \pm 2$, the SW curve (6.8) at this point is $\mathrm{e}^{-Y}+\mathrm{e}^{Y}+\widetilde{X}^{m} \pm 2=0$. At $\mathrm{e}^{-Y}=\mp 1$ and $\widetilde{X}=0$, the curve has an $A_{m-1}$ singularity

$$
Y^{2}+\widetilde{X}^{m}=0
$$

This is nothing but the curve at the Argyres-Douglas point in the $\mathrm{SU}(m)$ super-YangMills [33, 34, 35] if $m>2$, and it is a massless monopole point if $m=2$. The world-sheet CFT becomes singular precisely when some particles become massless in the space-time theory - from the string theory point of view, these particles are wrapped D-branes. This is the phenomenon which we already encountered before. For example, a massless charged hypermultiplet at type-IIB conifold singularity [36] and enhanced gauge symmetry (massless W-boson) at type-IIA $A D E$ singularity without B-field [37. Note that our LSM description is in type-IIA string theory and the singularity is the $A_{1}$ case of [37]. However, by tensoring with the minimal model and taking an orbifold, we see the phenomenon similar to [36] (it is equivalent for $m=2$ ).

This analysis of course generalizes to NS5-branes of type $\Gamma_{m}$, in which case we get Argyres-Douglas points of $\mathcal{N}=2$ super-Yang-Mills theory with gauge group $\Gamma_{m}$. In ref. [38] it was suggested that certain $\mathcal{N}=2 d=4$ SCFTs fall into the ADE classification. The present discussion relates this to the ADE classification of NS5-branes, which in turn is equivalent to the ADE classification of $\mathrm{SU}(2)$ modular invariants [19].

\subsection{Supergravity description of the coulomb branch}

It is not difficult to find the supergravity solution corresponding to the above one-parameter family of CFTs. We start with the LSM realization and integrate out the vector multiplets classically. This yields a non-linear sigma-model with a kählerian target space. The metric is

$$
\mathrm{d} s^{2}=2 f_{s}(r) d r^{2}+\frac{r^{2}}{2 f_{s}(r)}(\mathrm{d} \psi-\cos \theta \mathrm{d} \phi)^{2}+\frac{r^{2}}{2}\left(\mathrm{~d} \theta^{2}+\sin ^{2} \theta \mathrm{d} \phi^{2}\right)
$$

where

$$
f_{s}(r)=\frac{3 r^{2}-2 s}{2\left(r^{2}-s\right)}+\frac{2 r^{2}}{k}, \quad s=\operatorname{Re} t .
$$

The variable $\psi$ has period $2 \pi$. The variable $r$ ranges from $\sqrt{s}$ to $+\infty$ if $s>0$, and from 0 to $+\infty$ if $s \leq 0$.

\footnotetext{
${ }^{3}$ We can neglect the first $\mathrm{U}(1)$ since the corresponding vector multiplet is always heavy via mixing with $P$.
} 
The first thing to note about this metric is that it is non-singular for $s>0$ and has a curvature singularity at $r=0$ for $s \leq 0$. This means that for $s \leq 0$ quantum effects on the world-sheet are large, and one cannot use the non-linear sigma-model to describe the infrared physics of the LSM. In fact, even if $s$ is positive but small, the curvature is large near $r=\sqrt{s}$. Thus the semi-classical treatment of the world-sheet is valid only for $s$ large and positive. We therefore restrict ourselves to this regime.

Second, it is easy to see that topologically, and even algebro-geometrically, the manifold with the above metric is a copy of $T^{*} \mathbb{C P}^{1}$. The parameter $s$ is proportional to the area of the holomorphic 2-sphere which generates the second homology of $T^{*} \mathbb{C P}^{1}$.

Third, the above metric is not Ricci flat, so to determine the IR limit of the LSM we must figure out what it flows to. Since the target space is $T^{*} \mathbb{C P}^{1}$, the first thing that comes to mind is that the end-point of the flow is either a Eguchi-Hanson space, or a two-center Taub-NUT space, the two well-known Ricci flat metrics on $T^{*} \mathbb{C P}^{1}$. However, neither of them can be the end-point of the RG flow for our LSM. The Taub-NUT metric has a $\mathrm{U}(1) \times \mathrm{U}(1)$ isometry, while our metric (and therefore the whole RG trajectory) has a $\mathrm{U}(2)$ isometry. The Eguchi-Hanson metric has the right isometry, and in fact near the zero section the above metric is identical to Eguchi-Hanson. But their behaviors at infinity are quite different: while the Eguchi-Hanson metric is asymptotically locally euclidean (ALE), our metric is not. Rather, for fixed $r$ we have a squashed $\mathbb{R P}^{3}$, with the squashing increasing with $r$. In this respect our metric resembles the two-center Taub-NUT metric, except that the curvature of our metric falls off slower than in the Taub-NUT case.

Since the Eguchi-Hanson metric is the only kählerian Ricci flat metric with U(2) isometry, we conclude that the end-point cannot be Ricci flat. Rather, it is described by a kählerian dilatonic solution to the beta-function equations. Using the method of ref. [16], it is easy to show that the most general such solution with $\mathrm{U}(2)$ isometry and the asymptotics as in eq. (6.17) is given by eqs. (3.8) and eq. (3.9), but with $g_{2}(Y)$ replaced with

$$
\tilde{g}_{2}(Y)=\frac{1}{2 m} \frac{\frac{Y}{m}}{e^{-(Y-\sigma) / m}\left(1-\frac{\sigma}{m}\right)-1+\frac{Y}{m}} .
$$

In addition, $Y$ now ranges from $\sigma$ to $+\infty$.

The parameter $\sigma>0$ measures the area of $\mathbb{C P}^{1}$. Since the periods of the Kähler form are not affected by the RG flow in world-sheet perturbation theory, we must set $\sigma=s$. As discussed above, this derivation is valid for $s \gg 0$. Of course, world-sheet instantons can correct the relation between $s$ and $\sigma$, but these corrections are exponentially small for large $s$.

After tensoring with an $\mathcal{N}=2$ minimal model and performing T-duality, one gets a six-dimensional SUGRA background which is identical to the one found in refs. [12, 13. The parameter $k$ in ref. [12] is related to our $s$ as follows:

$$
k=\left(\frac{s}{m}-1\right) \exp \left(\frac{s}{m}\right) .
$$

This confirms that changing $k$ corresponds to moving on the Coulomb branch of the fivebrane theory. However, supergravity is a good description everywhere only for $k$ large 
and positive, i.e. when the size of $\mathbb{C P}^{1}$ is large. Here "good description" has the following meaning: although the supergravity solution has a singularity for all $k$, one can perform a T-duality which converts it to an (orbifold of) the product of an $\mathcal{N}=2$ minimal model and a smooth four-dimensional dilatonic background. It is the latter background which can be treated in the supergravity approximation.

As discussed above, there is also a good "global" interpretation of the solution with $k=-1$ : it corresponds to five-branes at the origin of the Coulomb branch. For other values of $k$ supergravity can be used only away from the singular locus containing five-branes.

\section{Compactification on $\mathbb{C P}^{2}$}

In this section we discuss type-IIB NS five-branes wrapped on $\mathbb{C P}^{2}$ in a similar manner. As discussed above, the naive expectation is that the low-energy theory reduces to $(2,2)$ super-Yang-Mills.

The quantity we would like to compute is again the mass of A-branes in the LG model eq. (5.21). It is given by the following period integral:

$$
\Pi=\int e^{Z} \mathrm{~d} Y_{1} \mathrm{~d} Y_{2} \mathrm{~d} Z \mathrm{~d} X \mathrm{~d} X_{2} \mathrm{~d} X_{3} \exp (-W) .
$$

Making a change of variables

$$
X_{i}=\mathrm{e}^{-m q_{i} Z} \widetilde{X}_{i}, \quad i=1,2,3,
$$

and integrating over $Z$, we find that the period integral is the same as for a Calabi-Yau 4-fold defined by the equation

$$
\mathrm{e}^{-Y_{1}}+\mathrm{e}^{-Y_{2}}+\mathrm{e}^{Y_{1}+Y_{2}}+W_{\Gamma_{m}}\left(\widetilde{X}_{i}\right)=0
$$

This was also obtained in ref. 39, 40 as the local mirror of a Calabi-Yau 4-fold with ADE singularity along $\mathbb{C P}^{2}$.

While in the $4 \mathrm{~d}$ case the mass of BPS states was interpreted in terms of a prepotential, in the $2 \mathrm{~d}$ case one can interpret it in terms of an effective superpotential [39, 40, 41]. The BPS states we are discussing are D4-branes wrapped on special lagrangian 4-cycles in a Calabi-Yau 4-fold $X$. In the low-energy 2d field theory they should be thought of as kinks interpolating between different vacua. As explained in ref. [41], the vacua are labelled by the periods of the 4-form flux $G$ on $X$. Indeed, since a D4-brane is magnetically charged with respect to $G$, the VEV of $G$ jumps as one moves across the D4-brane. The jump is equal to the Poincaré dual of the cycle wrapped by the brane.

Possible choices of $G$ are constrained by the requirement of $(2,2)$ supersymmetry [ 42 . In the low-energy field theory these constraints can be interpreted as arising from F-terms which depend on complex and Kähler moduli [41]. Since complex structure moduli and Kähler moduli live in chiral and twisted chiral multiplets, respectively, there will be a superpotential depending on $G$ and complex structure moduli and a twisted superpotential depending on $G$ and Kähler moduli. We are only concerned with the former. It is given by

$$
W=\frac{1}{2 \pi} \int \Omega \wedge G
$$


where $\Omega$ is the holomorphic 4-form on the Calabi-Yau (it is defined up to a constant factor). In our case $\Omega$ is given by

$$
\widetilde{X}_{1} \mathrm{~d} Y_{1} \mathrm{~d} Y_{2} \mathrm{~d} \widetilde{X}_{2} \mathrm{~d} \widetilde{X}_{3}
$$

Then it is easy to see that the BPS central charge of a special lagrangian brane is given by the change in this superpotential as one goes across the corresponding kink.

If we undo mirror symmetry, Käler and complex structure moduli, as well as ordinary and twisted superpotentials, are exchanged. Thus the superpotential we have written down maps to a twisted superpotential for the low-energy theory of type-IIB NS fivebranes wrapped on $\mathbb{C P}^{2}$. This twisted superpotential is induced by world-sheet instanton effects. One may ask if this twisted superpotential can be interpreted in terms of $(2,2)$ super-Yang-Mills, or is it an LST effect. This issue is discussed in the next section.

Finally, let us consider the following one-parameter family of deformations of the CY geometry (7.1)

$$
\mathrm{e}^{-Y_{1}}+\mathrm{e}^{-Y_{2}}+\mathrm{e}^{Y_{1}+Y_{2}}+W_{\Gamma_{m}}\left(\widetilde{X}_{i}\right)=\mu
$$

It corresponds to replacing $\mathrm{LSM}_{9 m}^{(3)} / \mathbb{Z}_{3}$ in $(5.19)$ by the IR limit of the following gauge theory

$$
\begin{array}{cccccc} 
& \Phi_{1} & \Phi_{2} & \Phi_{3} & Q & P \\
\mathrm{U}(1) & 1 & 1 & 1 & 0 & \text { shift } \\
\mathrm{U}(1) & 1 & 1 & 1 & -3 & 0
\end{array}
$$

with the orbifold group $\mathbb{Z}_{m}^{\text {diag }}$ acting as $\Phi_{i} \rightarrow \Phi_{i}, Q \rightarrow \mathrm{e}^{\frac{2 \pi i}{m}} Q$ and $P \rightarrow P$. The deformation parameter $\mu$ corresponds to $\mathrm{e}^{t / 3}$ where $t$ is the FI-Theta parameter of the second $\mathrm{U}(1)$. One can write down the corresponding one-parameter family of supergravity solutions generalizing eq. (4.9). It is obtained by replacing $g_{3}(Y)$ with

$$
\tilde{g}_{3}(Y)=\frac{Y^{2}}{3} \frac{e^{2 Y /(3 m)}}{\int_{\sigma}^{Y} t^{2} e^{2 t /(3 m)} d t},
$$

where $\sigma>0$, and restricting the range of $Y$ to $[\sigma,+\infty)$. This family describes a onedimensional submanifold of the Coulomb branch defined by the equations $u_{1}=\ldots=u_{\ell}=$ 0 . One can also think of it as describing $m$ NS5-branes wrapping the exceptional divisor of a noncompact Calabi-Yau 3-fold which is a crepant resolution of a $\mathbb{C}^{3} / \mathbb{Z}_{3}$ singularity. Note that taking $\sigma>0$ resolves the $\mathbb{Z}_{3}$ orbifold singularity present in eq. (4.9).

\section{Relation to Super-Yang-Mills theories}

The supergravity solution (4.2) preserves $1 / 4$ supersymmetry [12], so the effective field theory in four dimensions has $\mathcal{N}=2$ supersymmetry. What is this theory? The naive approach is to replace the theory of NS5-branes with its low-energy limit, $\mathcal{N}=2 d=6$ super-Yang-Mills theory, and then perform the Kaluza-Klein reduction. This procedure yields $\mathcal{N}=2 d=4$ super-Yang-Mills with the same gauge group as in six dimensions. However, since the decoupled theory on the NS5-branes is a non-local Little String Theory 
rather than a field theory, care is needed to make sense of this result. A difficulty arises when one tries to compute the $4 \mathrm{~d}$ gauge coupling. Naively, it is given by

$$
g_{4}^{2}=\frac{g_{6}^{2}}{4 \pi R^{2}}
$$

where $4 \pi R^{2}$ is the area of $\mathbb{C P}^{1}$. But the area of $\mathbb{C P}^{1}$ in the supergravity solution depends on $Y$, so one must specify at which $Y$ it must be evaluated. Usually, we measure the size at infinity in space, $Y=+\infty$, but then the area of $\mathbb{C P}^{1}$ is infinite too, which gives zero $4 \mathrm{~d}$ coupling. Another way to put the question is to ask what is the size of $\mathbb{C P}^{1}$ on which the LST is compactified. In ordinary field theory, there is always a definite answer, but in the present case, since $R(Y) \rightarrow+\infty$ for $Y \rightarrow \infty$, the situation is less clear.

Following ref. [11], we interpret the $Y$-dependence of various quantities as the dependence on the energy scale $E$ in the following way:

$$
E \sim \mathrm{e}^{-\Phi(Y)} .
$$

This relation is motivated by the way the mass of the W-boson scales with $g_{s t}=e^{\Phi}$. We would like to have a region of $Y$ that corresponds to high energies in $4 \mathrm{~d}$ physics but low energy compared to string scale and Kaluza-Klein scale, where the 4d gauge coupling is small. Since the $6 \mathrm{~d}$ gauge coupling is $g_{6}^{2}=2(2 \pi)^{3}$ and the volume of $\mathbb{C P}^{1}$ is $4 \pi R^{2}=2 \pi Y$, the $4 \mathrm{~d}$ gauge coupling at $Y$ is $g_{4}^{2}=2(2 \pi)^{3} / 2 \pi Y=2(2 \pi)^{2} / Y$. It is small at large $Y$ where the dilaton behaves as $\Phi(Y) \simeq-Y / 2 m+\Phi_{0}$. In such a region, the $4 \mathrm{~d}$ gauge coupling depends on the energy scale $E$ as

$$
\frac{8 \pi^{2}}{g_{4}^{2}} \sim Y \sim 2 m \log \left(E / \mathrm{e}^{-\Phi_{0}}\right) .
$$

This is the correct running of the gauge coupling, with the $4 \mathrm{~d}$ dimensional transmutation scale being

$$
\Lambda=\mathrm{e}^{-\Phi_{0}} .
$$

To extract just the super-Yang-Mills, we need $\Lambda$ to be smaller than the string scale (which is 1 ) and the Kaluza-Klein scale (which is of order $1 / 4 \pi R^{2} \sim 1 / 2 \pi Y$ ). This requires $e^{\Phi_{0}} \gg 1$. Thus the string coupling is extremely large in the relevant region of small $Y$.

Alternatively, we may go away from the origin of the Coulomb branch, so that the logarithmic running of $g_{4}^{2}$ stops at the scale of the Coulomb branch VEV $v$. This means that we must consider the one-parameter family of supergravity solutions ([.18). We identify the Coulomb branch VEV with the mass of a W-boson which is represented by a D-brane. Obviously, this D-brane must be sitting at the locus where its mass is minimized and the string coupling is maximized, i.e. at $Y=\sigma$. Then its mass is going to be of order $\exp (-\Phi(\sigma))$. The argument above can be repeated in this set-up: energy $E$ is replaced by the VEV $v \sim \exp (-\Phi(\sigma))$, and we end up with the same conclusion that decoupling requires $e^{\Phi_{0}} \gg 1$.

Thus the theory of wrapped five-branes reduces to pure $\mathcal{N}=2$ super-Yang-Mills only when the string coupling is taken to be very large. In this regime our world-sheet description is not applicable, and one has to use the S-dual description in terms of D5-branes wrapped 
on $\mathbb{C P}^{1}$. This is completely analogous to ref. [11. On the other hand, if one insists on working at weak string coupling, then the gauge theory scale $\Lambda$ is comparable or larger than the string scale, and one is dealing with an LST in 4d, rather than with a gauge theory. Our world-sheet computation of the prepotential in section 6 nevertheless agrees on the nose with the Seiberg-Witten solution of $\mathcal{N}=2$ super-Yang-Mills. This happens because of a supersymmetric non-renormalization theorem, which states that the $N=2$ $d=4$ prepotential cannot depend on the VEVs of hypermultiplets. In our case, the dilaton is part of a hypermuliplet, and therefore the prepotential cannot depend on it.

One can try to perform a similar analysis for five-branes wrapped on $\mathbb{C P}^{2}$, although it is complicated by infrared divergences which are present already at the classical level. The classical mass of the $\mathrm{W}$-boson on the Coulomb branch is made of a finite contribution of order $v$ and a divergent self-energy of order $g_{2}^{2} L$, where $L$ is the infrared cut-off. The mass of a D-brane has a similar structure, with the constant contribution of order $\exp (-\Phi(\sigma))$. We see that to keep the regularized D-brane mass below the string scale, we must work in the regime $e^{\Phi(\sigma)} \gg 1$.

Finally, let us comment on the size of world-sheet instantons in the decoupling limit. In the $\mathbb{C P}^{1}$ case, world-sheet instantons precisely correspond to gauge theory instantons. This is because in the S-dual (D5-brane) picture string world-sheet becomes the world-volume of a D1-brane, and D1-branes bound to D5-branes are nothing but Yang-Mills instantons. Their effects are bound to survive the decoupling limit. This was explicitly demonstrated in ref. [30] in the geometric engineering picture. In the $\mathbb{C P}^{2}$ case, it appears that worldsheet instanton effects disappear in the decoupling limit. For simplicity, let us set $m=2$. Then according to ref. [39] one-instanton contribution is of order

$$
v^{-6} e^{-\frac{c}{g_{2}}}
$$

The mass scale $c$ is of order one in string units; its precise value is unimportant. We want to take the decoupling limit $g_{2} \rightarrow 0, v \rightarrow 0$, while keeping the strength of the $2 \mathrm{~d}$ interactions fixed. This means we must keep $g_{2} / v$ fixed. Clearly, in this limit world-sheet instanton effects disappear. Another way to argue this is to note [39] that the decoupled theory is unchanged if we replace $\mathbb{C P}^{2}$ by any other Hirzebruch surface. On the other hand, such a replacement will drastically change world-sheet instanton contributions. The disappearance of the world-sheet instanton effects in the decoupling limit seems to solve

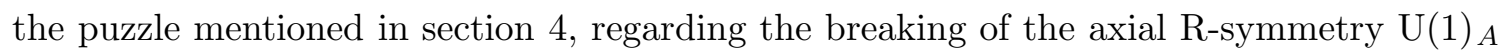
dow to $Z_{6 m}$.

In the 4-fold description of wrapped branes, it is easy to see that there are other effects which could lift the Coulomb branch. These are five-brane instantons wrapping both the $\mathbb{C P}^{2}$ base and the $A_{m-1}$ fiber. Similar five-brane instantons in M-theory compactified on a Calabi-Yau 4-fold are known to be responsible for the generation of the Affleck-HarveyWitten superpotential in $\mathcal{N}=2 d=3$ super-Yang-Mills 443, 44. Since IIA on a 4 -fold can be obtained from M-theory on a 4-fold by compactifying an extra circle, it is clear that five-brane instantons in type-IIA at finite string coupling also induce a superpotential on the Coulomb branch. It is not clear if such effects survive in the strict decoupling limit. 
An analogous question in field theory is whether the Affleck-Harvey-Witten superpotential survives dimensional reduction to $2 \mathrm{~d}$.

It is interesting to note that while world-sheet instantons induce a twisted superpotential depending on the Kähler moduli, five-brane instantons induce an ordinary superpotential for the T-duals of the Kähler moduli. Therefore it is impossible to write down a local action which includes both kinds of instantons. This is not so surprising, since five-brane instantons and world-sheet instantons are related by electric-magnetic duality and therefore are mutually non-local.

\section{Concluding remarks}

In this paper, we provided a world-sheet description of NS5-branes wrapped on $\mathbb{C P}^{1}$ in Eguchi-Hanson space and on $\mathbb{C P}^{2}$ in a non-compact CY 3-fold.

A striking aspect of the construction is its simplicity. Consider the origin of the moduli space of the $\mathbb{C P}^{1}$ compactification. Except for the well-understood minimal model, the nontrivial part of the world-sheet theory is the IR limit of a very simple linear sigma model U(1) gauge theory with two charge 1 matter fields plus a field $P$ that shifts under gauge transformations. Without the field $P$, the linear sigma model corrsponds to the non-linear sigma model on $\mathbb{C P}^{1}$ which is asymptotically free (the FI-theta parameter is dimensionally transmuted to a holomorphic scale parameter $\Lambda$ ) and has a mass gap. Introduction of the field $P$ corresponds to promoting the scale parameter $\Lambda$ to a field, since the dual of $P$ plays the role of the dynamical FI-Theta parameter. In other words, Re $P$ plays the role of the dynamical Weyl mode or "Liouville mode", which would appear when we quantize $2 \mathrm{~d}$ gravity coupled to this massive sigma model. The field $\operatorname{Im} P$ corresponds to adding a circle fibered over $\mathbb{C P}^{1}$. One may say that the present system is the world-sheet theory of a non-critical superstring on $\mathbb{R}^{3+1} \times\left[\mathbb{C P}^{1} \tilde{\times} \mathbb{S}^{1} \times \frac{\mathrm{SU}(2)_{m-2}}{\mathrm{U}(1)}\right] / \mathbb{Z}_{m}$, where $\tilde{\times}$ stands for a nontrivial fibration. With the orbifold action taken into account, the product $\mathbb{S}^{1} \times \frac{\mathrm{SU}(2)_{m-2}}{\mathrm{U}(1)}$ can be replaced by $\mathrm{SU}(2)_{m-2}$. Thus we are considering the non-critical superstring on

$$
\mathbb{R}^{3+1} \times \mathbb{C P}^{1} \tilde{\times} \mathrm{SU}(2)_{m-2} .
$$

The fibration $\mathbb{C P}^{1} \tilde{\times} \mathrm{SU}(2)_{m-2}$ is the one associated with the $\mathcal{N}=2$ twisting. We have shown that this describes the $\mathbb{C P}^{1}$ compactification of $m$ NS5-branes, at the origin of the moduli space. Moving away from the origin of the moduli space corresponds to adding massive matter, or performing a massive deformation of the minimal model $\frac{\mathrm{SU}(2)_{m-2}}{\mathrm{U}(1)}$. In the mirror LG model description it is more apparent that the scale parameter is promoted to a field.

The kählerian dilatonic backgrounds discovered in ref. [16] and used in this paper as building blocks, are very natural generalizations of the $2 \mathrm{~d}$ euclidean black hole and share many properties with the latter. For example, we have seen that they all arise as IR fixed points of an infinite sequence of linear sigma models. Since the $2 \mathrm{~d}$ black hole is integrable, it is natural to ask if its higher-dimensional generalizations also posess this property. We do not have strong evidence either against or in favor of this conjecture. We only performed the following integrability test. We have derived above the mirror description of 
our CFTs in terms of Landau-Ginzburg models. Given a Landau-Ginzburg model, it is quite straightforward to check whether it has non-trivial Bäcklund transformations depending on fields and their derivatives up to a certain order, see e.g. ref. [155. Therefore we tried to look for Bäcklund transformations in mirror LG models, with fermionic fields set to zero. In the case of the $2 \mathrm{~d}$ black hole the mirror LG model is a super-Liouville theory, whose integrability and Bäcklund transformations are well known. The LG model mirror to the $4 \mathrm{~d}$ generalization of the $2 \mathrm{~d}$ black hole is given by eqs. (5.3 5.4 with $a=0$. Unfortunately, since the Kähler potential is degenerate, the only way to make sense of this model is to regard it as a limit of the same model with $a \neq 0$. We have checked that in the neighborhood of $a=0$ the LG model defined by eqs. (5.3 5.4 does not have non-trivial Bäcklund transformations, if we allow dependence on derivatives up to fourth order.

\section{Acknowledgments}

We would like to thank Tohru Eguchi, Jaume Gomis, Aki Hashimoto and David Tong for useful discussions, and Juan Maldacena for explanations about the decoupling limit for wrapped five-branes. K. H. was supported in part by NSF-DMS 0074329 and by NSF-PHY 0070928. A. K. was supported in part by DOE grant DE-FG03-92-ER40701.

\section{References}

[1] G. 't Hooft, A planar diagram theory for strong interactions, Nucl. Phys. B 72 (1974) 461.

[2] A.M. Polyakov, String theory and quark confinement, Nucl. Phys. 68 (Proc. Suppl.) (1998) 1 hep-th/9711002; The wall of the cave, Int. J. Mod. Phys. A 14 (1999) 645 hep-th/9809057.

[3] N. Seiberg, New theories in six dimensions and matrix description of M-theory on $T^{5}$ and $T^{5} / \mathbb{Z}_{2}$, Phys. Lett. B 408 (1997) 98 hep-th/9705221.

[4] C.G. Callan, J.A. Harvey and A. Strominger, World sheet approach to heterotic instantons and solitons, Nucl. Phys. B 359 (1991) 611; Supersymmetric string solitons, hep-th/9112030.

[5] O. Aharony, M. Berkooz, D. Kutasov and N. Seiberg, Linear dilatons, ns5-branes and holography, J. High Energy Phys. 10 (1998) 004 hep-th/9808149.

[6] H. Ooguri and C. Vafa, Two-dimensional black hole and singularities of cy manifolds, Nucl. Phys. B 463 (1996) 55 hep-th/9511164.

[7] E. Witten, On string theory and black holes, Phys. Rev. D 44 (1991) 314. G. Mandal, A.M. Sengupta and S.R. Wadia, Classical solutions of two-dimensional string theory, Mod. Phys. Lett. A 6 (1991) 1685;

S. Elitzur, A. Forge and E. Rabinovici, Some global aspects of string compactifications, Nucl. Phys. B 359 (1991) 581.

[8] A. Giveon, D. Kutasov and O. Pelc, Holography for non-critical superstrings, J. High Energy Phys. 10 (1999) 035 hep-th/9907178.

[9] A. Giveon and D. Kutasov, Little string theory in a double scaling limit, J. High Energy Phys. 10 (1999) 034 hep-th/9909110; Comments on double scaled little string theory, 0 . High Energy Phys. 01 (2000) 023 hep-th/9911039. 
[10] T. Eguchi and Y. Sugawara, Modular invariance in superstring on Calabi-Yau n-fold with a-d-e singularity, Nucl. Phys. B 577 (2000) 3 hep-th/0002100.

[11] J.M. Maldacena and C. Núñez, Towards the large- $N$ limit of pure $N=1$ super Yang-Mills, Phys. Rev. Lett. 86 (2001) 588 hep-th/0008001.

[12] J.P. Gauntlett, N. Kim, D. Martelli and D. Waldram, Wrapped fivebranes and $N=2$ super Yang-Mills theory, Phys. Rev. D 64 (2001) 106008 hep-th/0106117.

[13] F. Bigazzi, A.L. Cotrone and A. Zaffaroni, $N=2$ gauge theories from wrapped five-branes, Phys. Lett. B 519 (2001) 269 hep-th/0106160.

[14] J. Gomis and J.G. Russo, $D=2+1 N=2$ Yang-Mills theory from wrapped branes, J. High Energy Phys. 10 (2001) 028 hep-th/0109177.

[15] J.P. Gauntlett, N.-W. Kim, D. Martelli and D. Waldram, Fivebranes wrapped on slag three-cycles and related geometry, J. High Energy Phys. 11 (2001) 018 hep-th/0110034.

[16] E. Kiritsis, C. Kounnas and D. Lüst, A large class of new gravitational and axionic backgrounds for four-dimensional superstrings, Int. J. Mod. Phys. A 9 (1994) 1361 hep-th/9308124.

[17] K. Hori and A. Kapustin, Duality of the fermionic 2d black hole and $N=2$ liouville theory as mirror symmetry, J. High Energy Phys. 08 (2001) 045 hep-th/0104202.

[18] K. Hori and C. Vafa, Mirror symmetry, hep-th/0002222.

[19] A. Cappelli, C. Itzykson and J.B. Zuber, Modular invariant partition functions in two-dimensions, Nucl. Phys. B 280 (1987) 445; The A-D-E classification of minimal and $A_{1}^{1}$ conformal invariant theories, Commun. Math. Phys. 113 (1987) 1.

D. Gepner and Z.-A. Qiu, Modular invariant partition functions for parafermionic field theories, Nucl. Phys. B 285 (1987) 423;

A. Kato, Classification of modular invariant partition functions in two-dimensions, Mod. Phys. Lett. A 2 (1987) 585.

[20] E. Calabi, Métriques kählériennes et fibrés holomorphes, Ann. Sci. École Norm. Sup. 12 (1979) 269.

[21] F. Ravanini and S.-K. Yang, Modular invariance in $N=2$ superconformal field theories, Phys. Lett. B 195 (1987) 202;

Z.-A. Qiu, Modular invariant partition functions for $N=2$ superconformal field theories, Phys. Lett. B 198 (1987) 497.

[22] J.M. Maldacena, G.W. Moore and N. Seiberg, Geometrical interpretation of D-branes in gauged WZW models, J. High Energy Phys. 07 (2001) 046 hep-th/0105038.

[23] J.M. Maldacena and C. Núñez, Supergravity description of field theories on curved manifolds and a no go theorem, Int. J. Mod. Phys. A 16 (2001) 822 hep-th/0007018.

[24] E. Witten, On the conformal field theory of the Higgs branch, J. High Energy Phys. 07 (1997) 003 hep-th/9707093.

[25] D.-E. Diaconescu and N. Seiberg, The coulomb branch of $(4,4)$ supersymmetric field theories in two dimensions, J. High Energy Phys. 07 (1997) 001 hep-th/9707158].

[26] E.J. Martinec, Algebraic geometry and effective lagrangians, Phys. Lett. B 217 (1989) 431. 
[27] C. Vafa and N.P. Warner, Catastrophes and the classification of conformal theories, Phys. Lett. B 218 (1989) 51.

[28] K. Hori, A. Iqbal and C. Vafa, D-branes and mirror symmetry, hep-th/0005247.

[29] A. Klemm, W. Lerche, P. Mayr, C. Vafa and N.P. Warner, Self-dual strings and $N=2$ supersymmetric field theory, Nucl. Phys. B 477 (1996) 746 hep-th/9604034.

[30] S. Katz, A. Klemm and C. Vafa, Geometric engineering of quantum field theories, Nucl. Phys. B 497 (1997) 173 hep-th/9609239.

[31] W. Lerche, On a boundary CFT description of nonperturbative $N=2$ Yang-Mills theory, hep-th/0006100.

[32] E. Witten, Phases of $N=2$ theories in two dimensions, Nucl. Phys. B 403 (1993) 159 hep-th/9301042.

[33] P.C. Argyres and M.R. Douglas, New phenomena in $\mathrm{SU}(3)$ supersymmetric gauge theory, Nucl. Phys. B 448 (1995) 93 hep-th/9505062.

[34] P.C. Argyres, M. Ronen Plesser, N. Seiberg and E. Witten, New $N=2$ superconformal field theories in four dimensions, Nucl. Phys. B 461 (1996) 71 hep-th/9511154.

[35] T. Eguchi, K. Hori, K. Ito and S.-K. Yang, Study of $n=2$ superconformal field theories in 4 dimensions, Nucl. Phys. B 471 (1996) 430 hep-th/9603002.

[36] A. Strominger, Massless black holes and conifolds in string theory, Nucl. Phys. B 451 (1995) 96 hep-th/9504090.

[37] P.S. Aspinwall, Enhanced gauge symmetries and K3 surfaces, Phys. Lett. B 357 (1995) 329 hep-th/9507012.

[38] T. Eguchi and K. Hori, $N=2$ superconformal field theories in 4 dimensions and a- $d$-e classification, hep-th/9607125.

[39] W. Lerche, Fayet-iliopoulos potentials from four-folds, J. High Energy Phys. 11 (1997) 004 hep-th/9709146.

[40] P. Kaste, On the twisted chiral potential in 2d and the analogue of rigid special geometry for 4-folds, J. High Energy Phys. 06 (1999) 021 hep-th/9904218.

[41] S. Gukov, C. Vafa and E. Witten, CFT's from Calabi-Yau four-folds, Nucl. Phys. B 584 (2000) 69 hep-th/9906070, erratum ibid. B 608 (2000) 477.

[42] K. Becker and M. Becker, M-theory on eight-manifolds, Nucl. Phys. B 477 (1996) 155 hep-th/9605053.

[43] E. Witten, Non-perturbative superpotentials in string theory, Nucl. Phys. B 474 (1996) 343 hep-th/9604030.

[44] S. Katz and C. Vafa, Geometric engineering of $N=1$ quantum field theories, Nucl. Phys. B 497 (1997) 196 hep-th/9611090.

[45] A.V. Ziber, N.H. Ibragimov and A.B. Shabat, Equations of Liouville type, Soviet Math. Dokl. 20 (1979) 1183. 